\title{
A GLOBAL DIMENSION THEOREM FOR QUANTIZED BANACH ALGEBRAS
}

\author{
N. V. VOLOSOVA
}

\begin{abstract}
We prove that for a commutative quantized $(\stackrel{h}{\otimes}$ and $\stackrel{o}{\otimes})$ algebra with infinite spectrum, the maximum of its left and right global homological dimensions and, as a consequence, its homological bidimension are strictly greater than one. This result is a quantum analog of the global dimension theorem of A. Ya. Helemskii.
\end{abstract}

\section{INTRODUCTION}

The goal of this paper is to extend the so-called global dimension theorem (see 11, [12, Prop. V.2.21] and [25]) - one of the main results in the homological theory of Banach algebras - to quantized algebras. It asserts that the global (homological) dimension $\operatorname{dg} A$ of a commutative Banach algebra with infinite spectrum is strictly larger than one. As a consequence, we have a bound on the homological bidimension for the corresponding classes of quantized algebras.

The history of the homological theory of Banach algebras goes back to 1962 when H. Kamovitz [19] defined homology groups of a Banach algebra $A$ with coefficients in a bimodule $X-$ a Banach analog of Hochschild cohomology, one of the key concepts of homological algebra. In 1970, A. Ya. Helemskii 8, using such tools from pure algebra as resolutions and derived functors, proposed a more general approach to the homology of Banach algebras; at the same time, the notions of homological dimension of a module over an algebra and the global homological dimension of an algebra were introduced. It turned out that the homological theory of Banach algebras has a number of features not found in its purely-algebraic counterpart. One such result without a purely-algebraic analog is the global dimension theorem, proved by A.Ya. Helemskii in 1972 9 (a complete proof can be found in [11; see also [25] for a detailed exposition). By now, the estimate $\operatorname{dg} A>1$ has also been obtained for some other classes of Banach algebras; we also know that, under certain conditions, global dimension is "well-behaved" under projective tensor product 28 .

We shall prove a similar theorem for quantized algebras with an additional, compared to Banach algebras, structure: quantum norm; such algebras are studied in the so-called quantized functional analysis. That branch of mathematics emerged in the beginning of the 1980s when, in the papers by Haagerup, Paulsen, and Wittstock, the concept of completely bounded map was introduced 21. The term "quantized functional analysis" was used by E. Effros in 1986 4. There are two essentially equivalent approaches to what should be understood by a quantum norm on a linear space $E$. The former (to which we adhere in this paper) deals with a family of norms on the space of matrices with entries in $E$. That theory is developed in [2, 6, 22]. In the other approach, one works with only one norm, rather than a family, defined on a larger space, namely $\mathcal{F} \otimes E$, where $\mathcal{F}$ is the

2000 Mathematics Subject Classification. Primary 46M18; Secondary 46H05, 46J20.

Supported by the RFFI (Project No. 05-01-00982 and Project No. 08-01-00867). 
space of finite-dimensional operators on a separable infinite-dimensional Hilbert space. An exposition of the theory of quantum spaces from that point of view can be found in [16] and [17].

Quantum (rather than usual) norms and the corresponding bounded operators seem to be the tools of choice for a large class of problems. Some of them that do not have satisfactory solutions in the framework of classical functional analysis admit elegant solutions when reformulated in quantum-theoretic terms. We list a few examples:

1. If operators $\varphi: E_{1} \rightarrow F_{1}$ and $\psi: E_{2} \rightarrow F_{2}$ between operator spaces are completely bounded (i.e., bounded in the quantum sense), then the operator $\varphi \otimes \psi$ between the tensor products $E_{1} \dot{\otimes} E_{2}$ and $F_{1} \dot{\otimes} F_{2}$ is also completely bounded ([3], [17, Th. 2.4.2]). A similar assertion for simply bounded operators is not true.

2. Quantum spaces admit a tensor product, denoted $\stackrel{\circ}{\otimes}$, which has the following property: if $A$ and $B$ are von Neumann algebras, then the predual space $(A \bar{\otimes} B)_{*}$ of their von Neumann tensor product is isomorphic to $A_{*} \stackrel{\circ}{\otimes} B_{*}[5$. No such tensor product exists in classical functional analysis.

3. In quantized functional analysis (in contrast to the classical case) there is a convenient criterion for an algebra to be completely isomorphic to an operator algebra 1 .

Methods of quantized functional analysis can often be used to obtain "classical" results.

4. The well-known Halmos similarity problem was reduced to a problem in quantum analysis [20] and in that form was solved in the negative [24].

The next two examples are related to topological homology and show how productive the quantum versions of such notions as projectivity, amenability, etc., can be.

5. One of the remarkable results in the theory of Banach algebras is a theorem of Johnson: the group algebra $L_{1}(G)$ of a locally compact group $G$ is amenable if and only if $G$ is amenable 18 . For another important algebra related to $G$, the Fourier algebra $A(G)$, this is not true. But if $A(G)$ is viewed as a quantized algebra, then it is amenable precisely when $G$ is 27 .

6. A von Neumann algebra is Wedderburn if and only if its space module is projective in the quantum sense [14.

As we see, the quantum theory of Banach algebras differs significantly from its classical counterpart. Nevertheless, a number of classical results, including the global dimension theorem, do have quantum analogs.

1.1. Preliminaries. We recall some basic definitions and facts. Let $E$ be a linear space. A matrix norm on $E$ is a family of norms $\|\cdot\|_{n}, n=1,2, \ldots$, on the spaces $M_{n}(E)$ of $n \times n$ matrices with entries in $E$. We shall say that $E$ is a quantized space or simply a quantum space if a matrix norm is given on $E$ and that norm satisfies the so-called Ruan axioms (see, for example, [6]):

(1) For any $\mathbf{a} \in M_{n}(E)$ and $\alpha, \beta \in M_{n}$ we have $\|\alpha \mathbf{a} \beta\|_{n} \leq\|\alpha\|\|\mathbf{a}\|_{n}\|\beta\|$ (here the product is that of matrices, and the norms of $\alpha$ and $\beta$ are their norms as of linear operators on $\left.\mathbb{C}^{n}\right)$.

(2) For any $\mathbf{a} \in M_{n}(E), \mathbf{b} \in M_{m}(E)$ and the block-diagonal matrix $\mathbf{a} \oplus \mathbf{b} \in M_{n+m}$ we have $\|\mathbf{a} \oplus \mathbf{b}\|_{n+m}=\max \left\{\|\mathbf{a}\|_{n},\|\mathbf{b}\|_{m}\right\}$.

Notice that the spaces $M_{m, n}(E)$ of $m \times n$ matrices with entries in $E$ also acquire a norm. More precisely, we define the norm of a matrix in $M_{m, n}(E)$ to be the norm of the square matrix obtained from it by adding zero rows or columns. It is easy to see that the result does not depend on how this was done. 
A quantum space is said to be Banach if the underlying space $E$ is complete with respect to $\|\cdot\|_{1}$ (and therefore, the spaces $M_{n}(E)$ are complete with respect to $\|\cdot\|_{n}$ ).

For the sake of brevity, when referring to the norm of an element of $M_{n}(E)$ we shall write $\|\cdot\|$ instead of $\|\cdot\|_{n}$.

If $F$ is a closed subspace of a quantum space $E$, then the quotient $E / F$ is also a quantum space: it is endowed with a quantum norm by identifying $M_{n}(E / F)$ with $M_{n}(E) / M_{n}(F)$. With this assumption, if $E$ is Banach, then $E / F$ is also Banach.

The direct sum $E \oplus F$ of quantum spaces $E$ and $F$ can be made into a quantum space by identifying $M_{n}(E \oplus F)$ with $M_{n}(E) \oplus M_{n}(F)$ : for $\left(a_{i j}\right) \in M_{n}(E)$ and $\left(b_{i j}\right) \in M_{n}(F)$ one has $\left\|\left(\left(a_{i j}, b_{i j}\right)\right)\right\|=\max \left\{\left\|\left(a_{i j}\right)\right\|,\left\|\left(b_{i j}\right)\right\|\right\}$.

Next we recall the constructions of the Haagerup $(E \stackrel{h}{\otimes} F)$ and the operator space projective $(E \stackrel{o}{\otimes} F)$ tensor product of quantum Banach spaces $E$ and $F$.

Let $\mathbf{v}=\left(v_{i j}\right) \in M_{n, m}(E)$ and $\mathbf{w}=\left(w_{i j}\right) \in M_{m, n}(F)$. The symbol $\mathbf{v} \odot \mathbf{w}$ will denote a matrix in $M_{n}(E \otimes F)$ with elements $(v \odot w)_{i j}=\sum_{k=1}^{m} v_{i k} \otimes w_{k j}$. One can show that any matrix $\mathbf{u} \in M_{n}(E \otimes F)$ can be written as $\mathbf{u}=\mathbf{v} \odot \mathbf{w}, \mathbf{v} \in M_{n, m}(E)$, w $\in M_{m, n}(F)$ for some $m \in \mathbb{N}$. We set

$$
\|\mathbf{u}\|:=\inf \{\|\mathbf{v}\|\|\mathbf{w}\|\}
$$

where the infimum is taken over all such products. One can show that this is indeed a norm on $M_{n}(E \otimes F)$. The Banach space $M_{n}(E \stackrel{h}{\otimes} F)$ is defined as the completion of $M_{n}(E \otimes F)$ with respect to this norm.

Now let $\mathbf{v}=\left(v_{i j}\right) \in M_{k, l}(E)$ and $\mathbf{w}=\left(w_{i j}\right) \in M_{q, r}(F)$. The symbol $\mathbf{v} \otimes \mathbf{w}$ will denote the matrix in $M_{k \times q, l \times r}(E \otimes F)$ (in such matrices the rows and columns are indexed by double indices) with elements $(v \otimes w)_{(g s)(h t)}=v_{g h} \otimes w_{s t}$. Any matrix $\mathbf{u} \in M_{n}(E \otimes F)$ can be written as the product $\alpha(\mathbf{v} \otimes \mathbf{w}) \gamma$, where $\mathbf{v} \in M_{k, l}(E)$, w $\in M_{q, r}(F)$, and $\alpha \in M_{n, k \times q}, \gamma \in M_{l \times r, n}$ for some $k, l, q, r \in \mathbb{N}$. We now set

$$
\|\mathbf{u}\|:=\inf \{\|\alpha\|\|\mathbf{v}\|\|\mathbf{w}\|\|\gamma\|\},
$$

where the infimum is taken over all such products. One can show that this is indeed a norm on $M_{n}(E \otimes F)$. The Banach space $M_{n}(E \stackrel{o}{\otimes} F)$ is defined as the completion of $M_{n}(E \otimes F)$ with respect to this norm.

We remark that the norm on $E \stackrel{o}{\otimes} F$ is greater than or equal to that on $E^{\stackrel{h}{\otimes}} F[\underline{6}$.

Next we want to recall some definitions related to the operators considered in quantized functional analysis.

Suppose $E, F, G$ are quantum spaces. An operator $\varphi: E \rightarrow F$ is said to be completely bounded if $\sup _{n}\left\|\varphi_{n}\right\|<\infty$, where

$$
\varphi_{n}: M_{n}(E) \rightarrow M_{n}(F), \quad\left(x_{i j}\right) \mapsto\left(\varphi\left(x_{i j}\right)\right) .
$$

That supremum is called the completely bounded norm of $\varphi$ and is denoted $\|\varphi\|_{c b}$.

Let $c b(E, F)$ denote the space of completely bounded operators from $E$ to $F$. By identifying $M_{n}(c b(E, F))$, for each $n \in \mathbb{N}$, with the space $c b\left(E, M_{n}(F)\right)$ endowed with the completely bounded norm, we introduce a matrix norm on $c b(E, F)$ making the latter a quantum space. We remark that $c b(E, \mathbb{C})=E^{*}$; i.e., any bounded linear functional on a quantum space is automatically completely bounded. Moreover, its completely bounded norm equals its norm as of a functional on a normed space [6].

A bioperator $\mathcal{R}: E \times F \rightarrow G$ is said to be $\stackrel{h}{\otimes}$-bounded if the operators

$$
\mathcal{R}_{n}^{(h)}: M_{n}(E) \times M_{n}(F) \rightarrow M_{n}(G),\left(\left(v_{i j}\right),\left(w_{i j}\right)\right) \mapsto\left(u_{i j}=\sum_{k=1}^{n} \mathcal{R}\left(v_{i k}, w_{k j}\right)\right),
$$


satisfy the condition $\sup \left\|\mathcal{R}_{n}^{(h)}\right\|<\infty$. It is said to be $\stackrel{o}{\otimes}$-bounded if the operators

$$
\mathcal{R}_{n}^{(o)}: M_{n}(E) \times M_{n}(F) \rightarrow M_{n \times n}(G),\left(\left(v_{i j}\right),\left(w_{i j}\right)\right) \mapsto\left(u_{(i j)(k l)}=\mathcal{R}\left(v_{i k}, w_{j l}\right)\right),
$$

satisfy the condition $\sup _{n}\left\|\mathcal{R}_{n}^{(o)}\right\|<\infty$. Those suprema are called, respectively, the $\stackrel{h}{\otimes}$ norm and $\stackrel{\circ}{\otimes}$-norm $\mathcal{R}$, and are denoted $\|\mathcal{R}\|_{\mathscr{Q}}$ and $\|\mathcal{R}\|_{\otimes}$. It is not difficult to prove that any $\stackrel{h}{\otimes}$-bounded bioperator $\mathcal{R}$ is automatically $\stackrel{o}{\otimes}$-bounded and that $\|\mathcal{R}\|_{\otimes} \leq\|\mathcal{R}\|_{\otimes}$.

Similar to the classical case, one can show that the spaces $E \stackrel{h}{\otimes} F$ and $E \stackrel{o}{\otimes} F$ have the universal property with respect to the classes of $\stackrel{h}{\otimes}$ - and $\stackrel{o}{\otimes}$-bounded operators, respectively. Moreover, the completely bounded norm of the associated operator equals, respectively, the $\stackrel{h}{\otimes}$ - and $\stackrel{o}{\otimes}$-norm of the original bioperator [2].

We shall now consider quantized algebras. $\mathrm{A} \stackrel{h}{\otimes}-(\stackrel{o}{\otimes}-)$ algebra is defined as a quantum Banach space $A$ endowed with a $\stackrel{h}{\otimes}-(\stackrel{o}{\otimes}-)$ bounded multiplication bioperator

$$
m: A \times A \rightarrow A \text {. }
$$

We may assume that $\|m\|_{\mathscr{h}} \leq 1\left(\|m\|_{\otimes} \leq 1\right)$, since otherwise $A$ can be renormed by replacing $\|\cdot\|_{n}$ by $\|m\|_{\mathscr{Q}}\|\cdot\|_{n}\left(\|m\|_{\otimes}\|\cdot\|_{n}\right)$ for all $n \in \mathbb{N}$. Notice that any $\stackrel{h}{\otimes}$-algebra is also a $\stackrel{o}{\otimes}$-algebra. Henceforth, we shall use the symbol $\widetilde{\otimes}$ in place of the symbols for the Haagerup $(\stackrel{h}{\otimes})$ and operator space projective $(\stackrel{o}{\otimes})$ tensor products in those cases when the arguments are the same for $\stackrel{h}{\otimes}$ - and $\stackrel{o}{\otimes}$-algebras. It is not difficult to check that the unitalization $A_{+}=A \oplus \mathbb{C}$ of the $\widetilde{\otimes}$-algebra $A$ (with quantization determined by direct sum) is also a $\widetilde{\otimes}$-algebra with respect to the multiplication $(a+\lambda e)(b+\mu e)=$ $a b+\lambda b+\mu a+\lambda \mu e$, where $a, b \in A, \lambda, \mu \in \mathbb{C}$ and $e=(0,1) \in A \oplus \mathbb{C}$ is the adjoined unit.

A left $\widetilde{\otimes}$-module over the $\widetilde{\otimes}$-algebra $A$ is a quantum Banach space $X$ endowed with a $\widetilde{\otimes}$-bounded multiplication bioperator

$$
\dot{m}: A \times X \rightarrow X .
$$

If, in addition, $A$ has a unit $e$ and $e \cdot x=x$ for all $x \in X$, then $X$ is said to be a unital module. Morphisms in the category of left $\widetilde{\otimes}$-modules over $A$ (denoted $\mathbf{A}-\widetilde{\otimes}$-mod) are completely bounded operators which are at the same time morphisms of modules in the algebraic sense. A morphism of $\widetilde{\otimes}$-modules having a completely bounded right inverse will be called an admissible epimorphism.

A module $P$ in $\mathbf{A}-\widetilde{\otimes}$-mod is said to be projective if for any $A-\widetilde{\otimes}$-modules $X$ and $Y$, any $A$ - $\widetilde{\otimes}$-module morphism $\varphi: P \rightarrow X$ and any admissible epimorphism $\sigma: Y \rightarrow X$, there is an $A$ - $\widetilde{\otimes}$-module morphism $\psi: P \rightarrow Y$ making the diagram

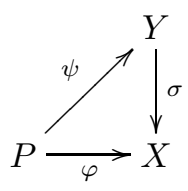

commute. For unital modules we have the following projectivity criterion: $X$ is projective in $\mathbf{A}-\widetilde{\otimes}-\mathbf{m o d}$ if and only if the canonical morphism $\pi: A \widetilde{\otimes} X \rightarrow X, a \otimes x \mapsto a \cdot x$, has a right inverse $\rho$ in $\mathbf{A}-\widetilde{\otimes}-\mathbf{m o d}$ (the proof of a similar criterion for Banach modules in $[12$ works in this case virtually without changes). In that case $\rho$ is called a coretraction $X$. 
The categories of right and two-sided $\widetilde{\otimes}$-modules over $A$ (denoted, respectively, $\widetilde{\otimes}$ mod-A and $\mathbf{A}-\widetilde{\otimes}-\mathbf{m o d}-\mathbf{A}$ ) as well as projective modules in those categories are defined similarly. For those classes of modules we have projectivity criteria similar to the one above. Henceforth, we shall mostly consider left modules, and projective modules in A$\widetilde{\otimes}$-mod will be simply called projective.

If $X \in \widetilde{\otimes}$-mod-A and $Y \in \mathbf{A}$ - $\widetilde{\otimes}$-mod, then the quantum space $X \underset{A}{\widetilde{\otimes}} Y$ is defined as the quotient of $X \widetilde{\otimes} Y$ by the closure of the linear span of elements of the form $x \cdot a \otimes y-x \otimes a \cdot y$, where $x \in X, y \in Y$ and $a \in A$. It is not difficult to show that $X \underset{A}{\widetilde{\otimes}} Y$ has a universal property with respect to the class of $\widetilde{\otimes}$-bounded balanced bilinear operators.

We shall also make use of the fact that the quotient of a $\widetilde{\otimes}$-algebra by a closed ideal or the quotient of a $\widetilde{\otimes}$-module by a closed submodule is again a $\widetilde{\otimes}$-algebra or a $\widetilde{\otimes}$-module of the same type (the proof of this fact is not difficult).

Let $A$ be a $\widetilde{\otimes}$-algebra. A complex of left $A-\widetilde{\otimes}$-modules

$$
0 \leftarrow X_{0} \leftarrow X_{1} \leftarrow X_{2} \leftarrow \cdots
$$

is said to be admissible if it is split in the category of quantum Banach spaces. In particular, an admissible complex is exact.

Let $X$ be a left $A-\widetilde{\otimes}$-module. A complex $\mathcal{X}$ of left $A$ - $\widetilde{\otimes}$-modules together with a morphism

$$
\varepsilon: X_{0} \rightarrow X
$$

is called a resolution of $X$ if the complex

$$
0 \leftarrow X \stackrel{\varepsilon}{\leftarrow} X_{0} \leftarrow X_{1} \leftarrow \cdots
$$

is admissible. A resolution $(\mathcal{X}, \varepsilon)$ is said to be projective if all modules in $\mathcal{X}$ are projective (in the category of left $A-\widetilde{\otimes}$-modules). The length of a shortest projective resolution of $X$ is called the homological dimension of $X$ over $A$ (denoted $\left.{ }_{A} \operatorname{dh} X\right)$.

Let

$$
0 \leftarrow X \stackrel{\varepsilon}{\leftarrow} X_{0} \stackrel{\partial_{0}}{\longleftarrow} X_{1} \stackrel{\partial_{1}}{\longleftarrow} X_{2} \stackrel{\partial_{2}}{\longleftarrow} \cdots
$$

be a projective resolution of a left $A-\widetilde{\otimes}$-module $X$. For each left $A$ - $\widetilde{\otimes}$-module $Y$ the group of extensions ${ }_{A} \operatorname{Ext}^{n}(X, Y)$ is defined as the $n$th cohomology group (in fact it is a linear space) of the complex

$$
0 \rightarrow{ }_{A} \operatorname{Hom}\left(X_{0}, Y\right) \stackrel{\partial_{0}^{*}}{\longrightarrow}{ }_{A} \operatorname{Hom}\left(X_{1}, Y\right) \stackrel{\partial_{1}^{*}}{\longrightarrow}{ }_{A} \operatorname{Hom}\left(X_{2}, Y\right) \stackrel{\partial_{2}^{*}}{\longrightarrow} \cdots .
$$

The result does not depend on the projective resolution of $X$, because all such resolutions are homotopically equivalent (cf. a similar construction for Banach modules in [12]). If $P$ is a projective left $A-\widetilde{\otimes}$-module, then, since it has a projective resolution of length $0,{ }_{A} \operatorname{Ext}^{n}(P, Y)=0$ for any left $A-\widetilde{\otimes}$-module $Y$ and any $n \geq 1$. Moreover, for any left $A$ - $\widetilde{\otimes}$-module $X$ we have

$$
{ }_{A} \operatorname{dh} X \leq n \Leftrightarrow{ }_{A} \operatorname{Ext}^{n+1}(X, Y)=0 \quad \text { for any left } A \text { - } \widetilde{\otimes} \text {-module } Y \text {. }
$$

We shall also make use of the following fact: let $0 \leftarrow X_{0} \leftarrow X_{1} \leftarrow X_{2} \leftarrow 0$ be a short admissible complex of left $A-\widetilde{\otimes}$-modules. Then for any left $A$ - $\widetilde{\otimes}$-module $Y$ we can construct a long exact sequence

$$
\begin{aligned}
0 & \rightarrow{ }_{A} \operatorname{Hom}\left(X_{0}, Y\right) \rightarrow{ }_{A} \operatorname{Hom}\left(X_{1}, Y\right) \rightarrow{ }_{A} \operatorname{Hom}\left(X_{2}, Y\right) \rightarrow{ }_{A} \operatorname{Ext}^{1}\left(X_{0}, Y\right) \\
& \rightarrow{ }_{A} \operatorname{Ext}^{1}\left(X_{1}, Y\right) \rightarrow{ }_{A} \operatorname{Ext}^{1}\left(X_{2}, Y\right) \rightarrow{ }_{A} \operatorname{Ext}^{2}\left(X_{0}, Y\right) \rightarrow \cdots .
\end{aligned}
$$

A proof of a similar result for Banach algebras is contained in [12, Th. III.4.4]; in the case of quantized algebras, the proof is virtually the same. 
The left global dimension of a $\widetilde{\otimes}$-algebra $A$ (denoted $\widetilde{\otimes} \operatorname{dg} A$ ) is the supremum of ${ }_{A} \mathrm{dh} X$ over all left $A$ - $\widetilde{\otimes}$-modules $X$.

Extension groups for right and two-sided $A-\widetilde{\otimes}$-modules $X$ and $Y$ (they are denoted, respectively, Ext ${ }_{A}^{n}(X, Y)$ and $\left.{ }_{A} \operatorname{Ext}_{A}^{n}(X, Y)\right)$, the homological dimension of a right $A-\widetilde{\otimes}$ module $X$ over $A$ (denoted $\operatorname{dh}_{A} X$ ), the homological dimension of an $A$ - $\widetilde{\otimes}$-bimodule $X$ over $A$ (denoted ${ }_{A} \mathrm{dh}_{A} X$ ), and the right global dimension of a $\widetilde{\otimes}$-algebra $A$ (denoted $\operatorname{dg}_{\tilde{\otimes}} A$ ) are defined similarly.

The homological bidimension of a $\widetilde{\otimes}$-algebra $A$ (denoted $\operatorname{db} A$ ) is defined as the dimension of $A_{+}$viewed as a bimodule over $A$, i.e., ${ }_{A} \mathrm{dh}_{A} A_{+}$. The left and right global dimensions of the $\widetilde{\otimes}$-algebra $A$ are related to its bidimension by the inequalities $\widetilde{\otimes} \operatorname{dg} A \leq \operatorname{db} A$ and $\operatorname{dg}_{\tilde{\otimes}} A \leq \operatorname{db} A[15$; the proof of this result repeats the similar proof in the classical setting [13, Th. 7.3.54] with obvious modifications.

The main result of this paper can now be stated as follows.

Theorem 1.1. Let $A$ be a commutative $\widetilde{\otimes}$-algebra with infinite spectrum. Then $\max \left\{\widetilde{\otimes} \operatorname{dg} A, \operatorname{dg}_{\tilde{\otimes}} A\right\} \geq 2$.

We remark that for $\stackrel{\circ}{\otimes}$-algebras the "classical" global dimension theorem, i.e., the inequality $\operatorname{dg} A \geq 2$ [11], remains the same because for such algebras the symmetry of the operator space projective tensor product implies that right $\stackrel{\circ}{\otimes}$-modules over $A$ are left $\stackrel{o}{\otimes}$-modules over $A^{o p}=A$, and therefore ${ }_{\otimes} \operatorname{dg} A=\operatorname{dg}_{\otimes} A=\max \left\{{ }_{\otimes} \operatorname{dg} A, \operatorname{dg}_{\otimes} A\right\}$.

From Theorem 1.1 and the above relations between the bidimension of an algebra and its left and right global dimensions we have:

Theorem 1.2. Let $A$ be a commutative $\widetilde{\otimes}$-algebra with infinite spectrum. Then $\mathrm{db} A \geq 2$.

1.2. Notation. Let $A$ be a commutative $\widetilde{\otimes}$-algebra with infinite Gelfand spectrum $\Omega$. Since $\tilde{\otimes} \operatorname{dg} A=\tilde{\otimes} \operatorname{dg} A_{+}$and $\operatorname{dg}_{\tilde{\otimes}} A=\operatorname{dg}_{\tilde{\otimes}} A_{+}$[15], henceforth we shall assume that $A$ is unital. The symbol $\hat{f}$ will denote the Gelfand transform of $f \in A$. The Shilov boundary of $A$ will be denoted $\partial A$.

Let $l^{\infty}$ denote the space of bounded complex sequences with quantization (it is said to be minimal) given by the matrix norm

$$
\left\|\left(x_{i j}\right)\right\|=\sup _{n}\left\|\left(x_{i j}(n)\right)\right\| \text {, where }\left(x_{i j}\right) \in M_{N}\left(l^{\infty}\right), N=1,2, \ldots
$$

(the Ruan axioms for this norm can easily be checked). Suppose $\left(s_{n}\right)$ is a convergent sequence in $\Omega$. Then the operators

$$
R: A \times l^{\infty} \rightarrow l^{\infty}, R(f, x)(n)=\hat{f}\left(s_{n}\right) x(n)
$$

and

$$
P: l^{\infty} \times A \rightarrow l^{\infty}, P(x, f)(n)=\hat{f}\left(s_{n}\right) x(n)
$$

are bilinear and $\stackrel{h}{\otimes}$ - (and therefore $\stackrel{o}{\otimes}$-) bounded, because for any $N \in \mathbb{N}$ and for all $\left(f_{i j}\right) \in M_{N}(A)$ and $\left(x_{i j}\right) \in M_{N}\left(l^{\infty}\right)$ we have

$$
\begin{aligned}
\left\|R_{N}^{(h)}\left(\left(f_{i j}\right),\left(x_{i j}\right)\right)\right\| & =\sup _{n}\left\|\left(\hat{f}_{i j}\left(s_{n}\right)\right)\left(x_{i j}(n)\right)\right\| \\
& \leq \sup _{n}\left\|\left(\hat{f}_{i j}\left(s_{n}\right)\right)\right\| \sup _{n}\left\|\left(x_{i j}(n)\right)\right\| \leq\left\|\left(f_{i j}\right)\right\|\left\|\left(x_{i j}\right)\right\|
\end{aligned}
$$

and similarly

$$
\left\|P_{N}^{(h)}\left(\left(x_{i j}\right),\left(f_{i j}\right)\right)\right\| \leq\left\|\left(f_{i j}\right)\right\|\left\|\left(x_{i j}\right)\right\| .
$$

Therefore $l^{\infty}$ is a $\widetilde{\otimes}$-bimodule over $A$ with scalar multiplication

$$
(f \cdot x)(n)=\hat{f}\left(s_{n}\right) x(n)=(x \cdot f)(n), f \in A, x \in l^{\infty} .
$$


It is also easy to show that the space $C_{0}\left(\mathbb{N}^{2}\right)$ with quantization given by the matrix norm

$$
\left\|\left(a_{i j}\right)\right\|=\sup _{m, n}\left\|\left(a_{i j}(m, n)\right)\right\|, \text { where }\left(a_{i j}\right) \in M_{N}\left(C_{0}\left(\mathbb{N}^{2}\right)\right), N=1,2, \ldots,
$$

is a $\widetilde{\otimes}$-bimodule over $A$ with scalar multiplication

$$
\begin{aligned}
& (f \cdot a)(m, n)=\hat{f}\left(s_{m}\right) a(m, n), \\
& (a \cdot f)(m, n)=\hat{f}\left(s_{n}\right) a(m, n), \quad f \in A, a \in C_{0}\left(\mathbb{N}^{2}\right) .
\end{aligned}
$$

Suppose $\omega \in \Omega$ is a limit point of $\left(s_{n}\right)$ and let $A_{\omega}$ be the maximal ideal of $A$ corresponding to $\omega$. Consider the closed ideal

$$
I=\left\{f \in A_{\omega}: \hat{f}\left(s_{n}\right)=0(n \in \mathbb{N})\right\}
$$

and the $\widetilde{\otimes}$-algebra

$$
B=A_{\omega} / I \text {. }
$$

It is clear that $B$ is a $\widetilde{\otimes}$-bimodule over $A$. It is easy to see that $B \widetilde{\otimes} B$ is also an $A$ - $\widetilde{\otimes}$-bimodule with scalar multiplication given by

$$
\begin{aligned}
& f \cdot([g] \otimes[h])=[f g] \otimes[h], \\
& ([g] \otimes[h]) \cdot f=[g] \otimes[h f], \quad f \in A, \quad g, h \in A_{\omega},
\end{aligned}
$$

and the operator

$$
\sigma_{0}: B \widetilde{\otimes} B \rightarrow B,[g] \otimes[h] \mapsto[g h]
$$

is a morphism of $A$ - $\widetilde{\otimes}$-bimodules. Consider the completely bounded operator

$$
\beta: B \widetilde{\otimes} B \rightarrow C_{0}\left(\mathbb{N}^{2}\right), \beta([g] \otimes[h])=\left(\hat{g}\left(s_{m}\right) \hat{h}\left(s_{n}\right)\right), \quad \text { where } \quad g, h \in A_{\omega} .
$$

It is well-defined and its image is contained in $C_{0}\left(\mathbb{N}^{2}\right)$ since $\left(s_{n}\right)$ converges to $\omega$. Moreover, $\beta$ is a morphism of $A$ - $\widetilde{\otimes}$-bimodules. It is easy to see that $\operatorname{ker} \beta \subset \operatorname{ker} \sigma_{0}$, because we can identify $\sigma_{0}([g] \otimes[h])$ with the sequence $\left(\hat{g}\left(s_{n}\right) \hat{h}\left(s_{n}\right)\right)$ of the "diagonal" elements of the double sequence $\beta([g] \otimes[h])$. Therefore $\sigma_{0}$ gives rise to a morphism of $A$ - $\widetilde{\otimes}$-bimodules

$$
\sigma: \widetilde{B} \rightarrow B, \text { where } \widetilde{B}=(B \widetilde{\otimes} B) / \operatorname{ker} \beta .
$$

Let $l_{e}$ denote $A_{\omega} \underset{A}{\widetilde{\otimes}} l^{\infty}$. It is well-defined because $A_{\omega}$ and $l^{\infty}$ are $\widetilde{\otimes}$-bimodules over $A$ and, in particular, $A_{\omega}$ is a right $A$ - $\widetilde{\otimes}$-module and $l^{\infty}$ is a left $A$ - $\widetilde{\otimes}$-module. Thus, $l_{e}$ is a $\widetilde{\otimes}$-bimodule over $A$ and, in particular, left $A$ - $\widetilde{\otimes}$-module. Now we can refine Theorem 1.1 as follows.

Theorem 1.3. Let $A$ be a commutative unital $\widetilde{\otimes}$-algebra with infinite spectrum. Then $\max \left\{\widetilde{\otimes} \operatorname{dg} A, \operatorname{dg}_{\tilde{\otimes}} A\right\} \geq 2$. More precisely, at least one of the following holds:

(1) There is a one-dimensional left $A-\widetilde{\otimes}$-module $X$ such that ${ }_{A} \operatorname{dh} X>1$.

(2) There is a one-dimensional right $A-\widetilde{\otimes}$-module $X$ such that $\mathrm{dh}_{A} X>1$.

(3) There is a convergent sequence $\left(s_{n}\right)$ of points in the Shilov boundary of A such that ${ }_{A} \mathrm{dh} l^{\infty}>1$. In particular,

$$
{ }_{A} \operatorname{Ext}^{2}\left(l^{\infty}, B \widetilde{\otimes} l_{e}\right) \neq 0 .
$$

A commutative unital $\widetilde{\otimes}$-algebra $A$ is said to be left weakly hereditary if all maximal ideals in $A$ are projective in $\mathbf{A}-\widetilde{\otimes}$-mod; it is said to be right weakly hereditary if they are all projective in $\widetilde{\otimes}$-mod-A; it is said to be weakly hereditary if they are all projective in both $\mathbf{A}-\widetilde{\otimes}-\mathbf{m o d}$ and in $\widetilde{\otimes}-\mathbf{m o d}-\mathbf{A}$. For the proof of Theorem 1.3 we need the following theorem, whose proof will be given later. 
Theorem 1.4. Let $A$ be a weakly hereditary commutative unital $\widetilde{\otimes}$-algebra with infinite spectrum $\Omega$. Then there is a sequence $\left(s_{n}\right)$ of pairwise distinct points in the Shilov boundary of $A$ converging to a point $\omega \in \partial A \backslash\left\{s_{n}\right\}_{n=1}^{\infty}$ such that

$$
\sigma: \widetilde{B} \rightarrow B
$$

is a retraction in the category of $A-\widetilde{\otimes}$-bimodules.

\section{A Reformulation of the Problem}

2.1. Reduction to the case of weakly hereditary commutative unital $\widetilde{\otimes}$-algebras. Suppose that the first and second parts of Theorem 1.3 are not true; i.e., ${ }_{A} \operatorname{dh} X \leq$ 1 for any one-dimensional left $A$ - $\widetilde{\otimes}$-module $X$ and $\operatorname{dh}_{A} Y \leq 1$ for any one-dimensional right $A-\widetilde{\otimes}$-module $Y$. For $s \in \Omega$ let $A_{s}$ be the corresponding maximal ideal in $A$. Then for the one-dimensional left $A$ - $\widetilde{\otimes}$-module $A / A_{s}$ and for any left $A$ - $\widetilde{\otimes}$-module $Y$ we have

$$
{ }_{A} \operatorname{Ext}^{2}\left(A / A_{s}, Y\right)=0 \text {. }
$$

The short admissible complex

$$
0 \leftarrow A / A_{s} \leftarrow A \leftarrow A_{s} \leftarrow 0
$$

gives rise to an exact sequence

$$
\begin{aligned}
0 & \rightarrow{ }_{A} \operatorname{Hom}\left(A / A_{s}, Y\right) \rightarrow{ }_{A} \operatorname{Hom}(A, Y) \rightarrow{ }_{A} \operatorname{Hom}\left(A_{s}, Y\right) \rightarrow{ }_{A} \operatorname{Ext}^{1}\left(A / A_{s}, Y\right) \\
& \rightarrow{ }_{A} \operatorname{Ext}^{1}(A, Y) \rightarrow{ }_{A} \operatorname{Ext}^{1}\left(A_{s}, Y\right) \rightarrow{ }_{A} \operatorname{Ext}^{2}\left(A / A_{s}, Y\right) \rightarrow \cdots .
\end{aligned}
$$

But ${ }_{A} \operatorname{Ext}^{1}(A, Y)=0$ since $A$ is a projective left $A$ - $\widetilde{\otimes}$-module and, as was mentioned before, ${ }_{A} \operatorname{Ext}^{2}\left(A / A_{s}, Y\right)=0$. The exactness of the sequence now implies that ${ }_{A} \operatorname{Ext}^{1}\left(A_{s}, Y\right)$ $=0$ for any left $A-\widetilde{\otimes}$-module $Y$. Therefore, $A_{s}$ is projective in $\mathbf{A}-\widetilde{\otimes}$-mod. Similarly, one can show that $A_{s}$ is projective in $\widetilde{\otimes}$-mod-A. Since this holds for all $s \in \Omega, A$ is a weakly hereditary $\widetilde{\otimes}$-algebra.

2.2. The entwining resolution. Let $A$ be a weakly hereditary commutative unital $\widetilde{\otimes}-$ algebra. By Theorem [1.4, there is a convergent sequence $\left(s_{n}\right)$ of pairwise distinct points on $\partial A$ with a limit point $\omega$ such that

$$
\sigma: \widetilde{B} \rightarrow B
$$

is a retraction in the category of $A-\widetilde{\otimes}$-bimodules. Define $l^{\infty}, l_{e}$, and $B$ as in Sec. 1 and set

$$
\mathcal{Q}=\left(A \widetilde{\otimes} l^{\infty}\right) \oplus l_{e}, \quad \mathcal{P}=\left(A \widetilde{\otimes} l_{e}\right) \oplus\left(A_{\omega} \widetilde{\otimes} l^{\infty}\right) .
$$

Lemma 2.1. There are short admissible complexes of left $A-\widetilde{\otimes}$-modules

$$
0 \rightarrow W \rightarrow \mathcal{Q} \rightarrow l^{\infty} \rightarrow 0 \quad \text { and } \quad 0 \rightarrow A_{\omega} \widetilde{\otimes} l_{e} \rightarrow \mathcal{P} \rightarrow W \rightarrow 0,
$$

where $W$ is a left $A-\widetilde{\otimes}$-module.

Proof. Consider the canonical morphism

$$
\varkappa: l_{e} \rightarrow l^{\infty}, \underset{A}{\otimes} x \mapsto f \cdot x, \text { where } f \in A_{\omega}, x \in l^{\infty} .
$$

Set

$$
\begin{array}{ll}
\partial_{-1}: & \mathcal{Q} \rightarrow l^{\infty}, \quad(f \otimes x, y) \mapsto f \cdot x+\varkappa(y), \\
\partial_{0}: & \mathcal{P} \rightarrow \mathcal{Q}, \quad(f \otimes y, g \otimes x) \mapsto(f \otimes \varkappa(y)-g \otimes x, g \underset{A}{\otimes x} x-f \cdot y), \\
\partial_{1}: & A_{\omega} \otimes l_{e} \rightarrow \mathcal{P}, \quad g \otimes y \mapsto(g \otimes y, g \otimes \varkappa(y)),
\end{array}
$$


where $f \in A, x \in l^{\infty}, y \in l_{e}, g \in A_{\omega}$. We now have well-defined morphisms of left $A-\widetilde{\otimes}$-modules. We claim that the sequence

$$
0 \leftarrow l^{\infty} \stackrel{\partial_{-1}}{\longleftarrow} \mathcal{Q} \stackrel{\partial_{0}}{\longleftarrow} \mathcal{P} \stackrel{\partial_{1}}{\longleftarrow} A_{\omega} \widetilde{\otimes} l_{e} \leftarrow 0
$$

is a resolution of the $A$ - $\widetilde{\otimes}$-module $l^{\infty}$. That $\mathcal{R}$ is a complex can be checked by direct calculations. It remains to find a contracting homotopy for this complex in the category of quantum Banach spaces.

Let $e$ be the unit in $A$. Then the maps

$$
\begin{array}{ll}
s_{-1}: & l^{\infty} \rightarrow \mathcal{Q}, \quad x \mapsto(e \otimes x, 0), \\
s_{0}: & \mathcal{Q} \rightarrow \mathcal{P}, \\
& (f \otimes x, y) \mapsto(-e \otimes((f-\hat{f}(\omega) e){\underset{A}{A} x}-e \otimes y,(\hat{f}(\omega) e-f) \otimes x), \\
s_{1}: & \mathcal{P} \rightarrow A_{\omega} \widetilde{\otimes} l_{e}, \quad(f \otimes y, g \otimes x) \mapsto(f-\hat{f}(\omega) e) \otimes y,
\end{array}
$$

where $f \in A, g \in A_{\omega}, y \in l_{e}, x \in l^{\infty}$, are completely bounded linear operators and it is not difficult to check that they yield a contracting homotopy for $\mathcal{R}$.

Thus, $\mathcal{R}$ is admissible and is a resolution of $l^{\infty}$. It is called the entwining resolution (cf. [12, Ch. V, $\S 2.1]$ ). Set $W=\operatorname{ker} \partial_{-1}=\operatorname{im} \partial_{0}$. Then we have the desired short admissible sequences

$$
0 \rightarrow W \rightarrow \mathcal{Q} \rightarrow l^{\infty} \rightarrow 0
$$

and

$$
0 \rightarrow A_{\omega} \widetilde{\otimes} l_{e} \rightarrow \mathcal{P} \rightarrow W \rightarrow 0 .
$$

The short admissible complex

$$
0 \rightarrow W \rightarrow \mathcal{Q} \rightarrow l^{\infty} \rightarrow 0
$$

gives rise to an exact sequence

$$
\cdots \rightarrow{ }_{A} \operatorname{Ext}^{1}\left(\mathcal{Q}, B \widetilde{\otimes} l_{e}\right) \rightarrow{ }_{A} \operatorname{Ext}^{1}\left(W, B \widetilde{\otimes} l_{e}\right) \rightarrow{ }_{A} \operatorname{Ext}^{2}\left(l^{\infty}, B \widetilde{\otimes} l_{e}\right) \rightarrow \cdots .
$$

It remains to show that ${ }_{A} \operatorname{Ext}^{1}\left(\mathcal{Q}, B \widetilde{\otimes} l_{e}\right)=0$ and ${ }_{A} \operatorname{Ext}^{1}\left(W, B \widetilde{\otimes} l_{e}\right) \neq 0$. Then it would follow from the exactness of the sequence that ${ }_{A} \operatorname{Ext}^{2}\left(l^{\infty}, B \widetilde{\otimes} l_{e}\right) \neq 0$, and Theorem 1.3 would be proved.

\section{Some Properties of PRojeCtive maximal ideals}

In this section we shall prove several properties of projective maximal ideals that are important for the rest of the paper.

Lemma 3.1. 1) Let $A$ be a commutative unital $\widetilde{\otimes}$-algebra and $\omega$ a point in the Shilov boundary of $A$ such that the corresponding maximal ideal $A_{\omega}$ is projective in $\mathbf{A}-\widetilde{\otimes}-\bmod$. Then $\overline{A_{\omega}^{2}}=A_{\omega}$.

2) Let $A$ be a commutative unital $\widetilde{\otimes}$-algebra and $\omega$ a point in the Shilov boundary of $A$ such that the corresponding maximal ideal $A_{\omega}$ is projective in $\widetilde{\otimes}-\mathbf{m o d}-\mathbf{A}$. Then $\overline{A_{\omega}^{2}}=A_{\omega}$.

The proof of this lemma is almost the same as the proof for left modules over Banach algebras [11, Lemmas 1.1 and 1.2] and is therefore omitted.

Lemma 3.2. 1) Let $A$ be a commutative unital $\widetilde{\otimes}$-algebra and $A_{\omega}$ a maximal ideal in $A$ which is projective in $\mathbf{A}-\widetilde{\otimes}-\mathbf{m o d}$ and such that $\overline{A_{\omega}^{2}}=A_{\omega}$. Then the image of any coretraction

$$
\rho: A_{\omega} \rightarrow A \widetilde{\otimes} A_{\omega}
$$

of $A_{\omega}$ is contained in $A_{\omega} \widetilde{\otimes} A_{\omega}$. 
2) Let $A$ be a commutative unital $\widetilde{\otimes}$-algebra, and $A_{\omega}$ a maximal ideal in $A$ which is projective in $\widetilde{\otimes}$-mod-A and such that $\overline{A_{\omega}^{2}}=A_{\omega}$. Then the image of any coretraction

$$
\rho: A_{\omega} \rightarrow A_{\omega} \widetilde{\otimes} A
$$

of $A_{\omega}$ is contained in $A_{\omega} \widetilde{\otimes} A_{\omega}$.

Proof. We prove 1). For any element of the form $f h$, where $f, h \in A_{\omega}$, we have

$$
\rho(f h)=f \rho(h) \in A_{\omega} \widetilde{\otimes} A_{\omega} .
$$

Since the linear span of such elements is dense in $A_{\omega}$ and $\rho$ is completely bounded, we have the desired assertion. 2) is proved similarly.

Definition 3.3. Let $\Omega$ be the spectrum of a commutative unital $\widetilde{\otimes}$-algebra $A$ and $\omega \in \Omega$. For $s \in \Omega \backslash\{\omega\}$ set

$$
K_{s}=\inf \left\{\|f\|: f \in A_{\omega}, \hat{f}(s)=1\right\} .
$$

Lemma 3.4. Let $A$ be a commutative unital $\widetilde{\otimes}$-algebra with spectrum $\Omega$ and $A_{\omega} a$ projective maximal ideal in $A$ such that $\overline{A_{\omega}^{2}}=A_{\omega}$. Then for any coretraction $\rho$ of $A_{\omega}$ and for each $s \in \Omega \backslash\{\omega\}$ we have $K_{s} \leq\|\rho\|_{c b}$.

Proof. We have separate proofs for $\stackrel{h}{\otimes}$ - and $\stackrel{o}{\otimes}$-algebras.

1. $\stackrel{h}{\otimes}$-algebra. Let $\rho$ be a coretraction of $A_{\omega}$. For each $f \in A_{\omega}$ we have $\rho(f) \in A_{\omega} \stackrel{h}{\otimes} A_{\omega}$; hence we may write $\rho(f)$ as

$$
\rho(f)=\lim _{n \rightarrow \infty}\left(g_{i j}^{(n)}\right) \odot\left(h_{i j}^{(n)}\right),
$$

where for all $n,\left(g_{i j}^{(n)}\right) \in M_{1, N}\left(A_{\omega}\right),\left(h_{i j}^{(n)}\right) \in M_{N, 1}\left(A_{\omega}\right)$ for some $N \in \mathbb{N}$ (depending on $n$ ), and

$$
\lim _{n \rightarrow \infty}\left\|\left(g_{i j}^{(n)}\right)\right\|\left\|\left(h_{i j}^{(n)}\right)\right\|=\|\rho(f)\| .
$$

Suppose $s \in \Omega \backslash\{\omega\}$. Consider the functional

$$
\chi_{s}: A_{\omega} \rightarrow \mathbb{C}, f \mapsto \hat{f}(s) .
$$

Since for all $f \in A_{\omega}$, we have $\hat{f}(s) \mid \leq 1 / K_{s}\|f\|$, it follows that $\left\|\chi_{s}\right\| \leq 1 / K_{s}$. Therefore, $\left\|\chi_{s}\right\|_{c b} \leq 1 / K_{s}$; i.e., for any matrix $\left(f_{i j}\right)$ with entries in $A_{\omega}$ we have

$$
\left\|\left(f_{i j}\right)\right\| \geq K_{s}\left\|\left(\hat{f}_{i j}(s)\right)\right\| .
$$

In particular, for all $n$,

$$
\left\|\left(g_{i j}^{(n)}\right)\right\|\left\|\left(h_{i j}^{(n)}\right)\right\| \geq\left\|\left(\hat{g}_{i j}^{(n)}(s)\right)\right\|\left\|\left(\hat{h}_{i j}^{(n)}(s)\right)\right\| K_{s}^{2} \geq\left\|\left(\hat{g}_{i j}^{(n)}(s)\right)\left(\hat{h}_{i j}^{(n)}(s)\right)\right\| K_{s}^{2} .
$$

Therefore,

$$
\|\rho(f)\|=\lim _{n \rightarrow \infty}\left\|\left(g_{i j}^{(n)}\right)\right\|\left\|\left(h_{i j}^{(n)}\right)\right\| \geq \lim _{n \rightarrow \infty}\left\|\left(\hat{g}_{i j}^{(n)}(s)\right)\left(\hat{h}_{i j}^{(n)}(s)\right)\right\| K_{s}^{2}=|\hat{f}(s)| K_{s}^{2} .
$$

We now have

$$
K_{s}^{2} \leq\|\rho(f)\| \leq\|\rho\|_{c b}\|f\|
$$

for any $f \in A_{\omega}$ such that $\hat{f}(s)=1$. Thus, $K_{s}^{2} \leq K_{s}\|\rho\|_{c b}$ and $K_{s} \leq\|\rho\|_{c b}$. 
2. $\stackrel{o}{\otimes}$-algebra. We write $\rho(f)$ in the form

$$
\rho(f)=\lim _{n \rightarrow \infty}\left(\alpha_{i j}^{(n)}\right)\left(\left(g_{i j}^{(n)}\right) \otimes\left(h_{i j}^{(n)}\right)\right)\left(\beta_{i j}^{(n)}\right),
$$

where for each $n,\left(g_{i j}^{(n)}\right) \in M_{l, k}\left(A_{\omega}\right),\left(h_{i j}^{(n)}\right) \in M_{p, t}\left(A_{\omega}\right),\left(\alpha_{i j}^{(n)}\right) \in M_{1, l \times p},\left(\beta_{i j}^{(n)}\right) \in$ $M_{k \times t, 1}$ for some $l, k, p, t \in \mathbb{N}$ (depending on $n$ ) and

$$
\lim _{n \rightarrow \infty}\left\|\left(\alpha_{i j}^{(n)}\right)\right\|\left\|\left(g_{i j}^{(n)}\right)\right\|\left\|\left(h_{i j}^{(n)}\right)\right\|\left\|\left(\beta_{i j}^{(n)}\right)\right\|=\|\rho(f)\| .
$$

The rest of the proof is similar to the $\stackrel{h}{\otimes}$-case.

This lemma yields the following analog of Cor. 1.5 in [11:

Corollary 3.5. Let $A$ be a commutative unital $\widetilde{\otimes}$-algebra with spectrum $\Omega$ and suppose that $\omega_{1}, \ldots, \omega_{n} \in \Omega$ are such that the corresponding ideals $A_{1}, \ldots, A_{n}$ are projective and $\overline{A_{m}^{2}}=A_{m}$ for $m=1, \ldots, n$. Then there is $K>0$ such that for any $s \in \Omega \backslash\left\{\omega_{1}, \ldots, \omega_{n}\right\}$ there exists $f \in A$ with $\hat{f}\left(\omega_{m}\right)=0$ for $m=1, \ldots, n, \hat{f}(s)=1$, and $\|f\|<K$.

\section{A trivial extension group}

Recall the situation described in Sec. 2] let $A$ be a weakly hereditary commutative unital $\widetilde{\otimes}$-algebra, and $\left(s_{n}\right)$ a sequence of pairwise distinct points in the Shilov boundary of $A$ converging to a point $\omega \in \partial A \backslash\left\{s_{n}\right\}_{n=1}^{\infty}$ and such that

$$
\sigma: \widetilde{B} \rightarrow B
$$

is a retraction in the category of $A$ - $\widetilde{\otimes}$-bimodules. We want to $\operatorname{show}_{A} \operatorname{Ext}^{1}\left(\mathcal{Q}, B \widetilde{\otimes} l_{e}\right)=0$.

Since $A \widetilde{\otimes} l^{\infty}$ is a projective left $A$ - $\widetilde{\otimes}$-module,

$$
{ }_{A} \operatorname{Ext}^{1}\left(A \widetilde{\otimes} l^{\infty}, B \widetilde{\otimes} l_{e}\right)=0,
$$

and it suffices to show that

$$
{ }_{A} \operatorname{Ext}^{1}\left(l_{e}, B \widetilde{\otimes} l_{e}\right)=0,
$$

because $\mathcal{Q}=\left(A \widetilde{\otimes} l^{\infty}\right) \oplus l_{e}$ and the functor Ext preserves direct sums.

Consider the short exact complex of left $A-\widetilde{\otimes}$-modules

$$
0 \rightarrow M \stackrel{i}{\hookrightarrow} A_{\omega} \widetilde{\otimes} l_{e} \stackrel{\pi_{e}}{\longrightarrow} l_{e} \rightarrow 0 \quad(\mathcal{C})
$$

where $\pi_{e}$ is determined by the condition $\pi_{e}(a \otimes b \underset{A}{\otimes} x)=a b \underset{A}{\otimes} x$ for all $a, b \in A_{\omega}, x \in l^{\infty}$, $M=\operatorname{ker} \pi_{e}$, and $i$ is the natural embedding.

Lemma 4.1. $\mathcal{C}$ is a short admissible complex.

Proof. Since $\omega$ is in the Shilov boundary of $A$, we have, by Sec. 3 , that $\overline{A_{\omega}^{2}}=A_{\omega}$ and $\rho\left(A_{\omega}\right) \subset A_{\omega} \widetilde{\otimes} A_{\omega}$ for any morphism of right $A$ - $\widetilde{\otimes}$-modules

$$
\rho: A_{\omega} \rightarrow A_{\omega} \widetilde{\otimes} A,
$$

which is a right inverse of the canonical morphism

$$
\pi: A_{\omega} \widetilde{\otimes} A \rightarrow A_{\omega} .
$$

Hence we can define

$$
\rho_{e}: l_{e} \rightarrow A_{\omega} \widetilde{\otimes} l_{e}, \rho_{e}=\rho \underset{A}{\widetilde{\otimes}} \mathbf{1}_{l^{\infty}} .
$$

The operator $\rho_{e}$ is well-defined because $\rho$ is a morphism of right $A$ - $\widetilde{\otimes}$-modules and is completely bounded. Moreover

$$
\pi_{e} \circ \rho_{e}(f \underset{A}{\otimes} x)=\pi \underset{A}{\otimes} \mathbf{1}_{l^{\infty}}(\rho(f) \underset{A}{\otimes} x)=f \underset{A}{\otimes} x \text { for } f \in A_{\omega}, x \in l^{\infty} .
$$

Therefore, $\rho_{e}$ is a right inverse of $\pi_{e}$, and the complex $\mathcal{C}$ is admissible. 
The admissible complex $\mathcal{C}$ gives rise to a long exact sequence

$$
\begin{aligned}
0 \rightarrow{ }_{A} \operatorname{Hom}\left(l_{e}, B \widetilde{\otimes} l_{e}\right) \stackrel{\pi_{e}^{*}}{\longrightarrow}{ }_{A} \operatorname{Hom}\left(A_{\omega} \widetilde{\otimes} l_{e}, B \widetilde{\otimes} l_{e}\right) \stackrel{i^{*}}{\rightarrow}{ }_{A} \operatorname{Hom}\left(M, B \widetilde{\otimes} l_{e}\right) \\
\rightarrow{ }_{A} \operatorname{Ext}^{1}\left(l_{e}, B \widetilde{\otimes} l_{e}\right) \rightarrow{ }_{A} \operatorname{Ext}^{1}\left(A_{\omega} \widetilde{\otimes} l_{e}, B \widetilde{\otimes} l_{e}\right) \rightarrow \cdots .
\end{aligned}
$$

Notice that ${ }_{A} \operatorname{Ext}^{1}\left(A_{\omega} \widetilde{\otimes} l_{e}, B \widetilde{\otimes} l_{e}\right)=0$ because the module $A_{\omega} \widetilde{\otimes} l_{e}$ is projective. Thus, to prove that ${ }_{A} \operatorname{Ext}^{1}\left(l_{e}, B \widetilde{\otimes} l_{e}\right)=0$ it suffices to show that $i^{*}$ is surjective.

Let

$$
\tau \widetilde{\otimes} \mathbf{1}_{l_{e}}: A_{\omega} \widetilde{\otimes} l_{e} \rightarrow B \widetilde{\otimes} l_{e}, L=\operatorname{ker}\left(\tau \widetilde{\otimes} \mathbf{1}_{l_{e}}\right),
$$

where $\tau$ is the natural projection to $B=A_{\omega} / I$.

Lemma 4.2. $L \subset M$.

Proof. The proof consists of several simple propositions.

Proposition 1. The linear span of the elements $\underset{A}{\underset{A}{\otimes}} x, f \in A_{\omega}, x \in c_{0}$, is dense in $l_{e}$.

It suffices to show that each $g{\underset{A}{\otimes}}_{y} y$, where $g \in A_{\omega}$ and $y \in l^{\infty}$, belongs to the closure of the linear span of the elements $\underset{A}{A} \underset{A}{\otimes}, f \in A_{\omega}, x \in c_{0}$. For $h, k \in A_{\omega}$ we have

$$
h k \underset{A}{\otimes} y=h \underset{A}{\otimes} k \cdot y,
$$

and $k \cdot y \in c_{0}$ for all $y \in l^{\infty}$. Since $\overline{A_{\omega}^{2}}=A_{\omega}$, the desired assertion follows from Lemma 3.1 .

Let $P^{n}: l^{\infty} \rightarrow l^{\infty}$ be the canonical projection to the $n$th component and $Q^{k}=$ $\sum_{n=1}^{k} P^{n}$. Define

$$
\begin{aligned}
& Q_{e}^{k}: l_{e} \rightarrow l_{e}, Q_{e}^{k}=\mathbf{1}_{A_{\omega}} \underset{{ }_{\otimes}}{\widetilde{A}} Q^{k}, \\
& P_{e}^{n}: l_{e} \rightarrow l_{e}, P_{e}^{n}=\mathbf{1}_{A_{\omega}} \underset{A}{\widetilde{\otimes}} P^{n} .
\end{aligned}
$$

Proposition 2. $\lim _{k \rightarrow \infty} Q_{e}^{k} z=z$ for any $z \in l_{e}$.

By Proposition 1, it suffices to prove the assertion for elements of the form $f \underset{A}{\otimes} x$, where $f \in A_{\omega}, x \in c_{0}$. But for such elements it is obvious because $\lim _{k \rightarrow \infty} Q^{k} x=x$.

Proposition 3. Suppose $f \in A_{\omega}$ and $\hat{f}\left(s_{n}\right)=0$ for some $n \in \mathbb{N}$. Then $\underset{A}{\underset{A}{\otimes}} e_{n}=0$.

Choose $h \in A_{\omega}$ such that $\hat{h}\left(s_{n}\right)=1$. Then

$$
f \underset{A}{\otimes} e_{n}=f \underset{A}{\otimes} h \cdot e_{n}=f h \underset{A}{\otimes} e_{n}=h f \underset{A}{\otimes} e_{n}=h \underset{A}{\otimes} f \cdot e_{n}=0 .
$$

Suppose now that $q^{n}=\underset{A}{\underset{A}{\otimes} e_{n}}$ for some $f \in A_{\omega}$ such that $\hat{f}\left(s_{n}\right)=1$. By Proposition 3 , $q^{n}$ does not depend on the choice of $f$.

Proposition 4. The space $L$ is the closed linear span of the elements $f \otimes q^{n}$, where $f \in I$ and $n \in \mathbb{N}$.

It is clear that $f \otimes q^{n} \in L$ for all $f \in I, n \in \mathbb{N}$. For $u \in L$ we have

$$
\left(\tau \widetilde{\otimes} \mathbf{1}_{l_{e}}\right)\left(\mathbf{1}_{A_{\omega}} \widetilde{\otimes} P_{e}^{n}\right) u=\left(\tau \widetilde{\otimes} P_{e}^{n}\right) u=\left(\mathbf{1}_{B} \widetilde{\otimes} P_{e}^{n}\right)\left(\tau \widetilde{\otimes} \mathbf{1}_{l_{e}}\right) u=0 .
$$

Therefore, $\left(\mathbf{1}_{A_{\omega}} \widetilde{\otimes} P_{e}^{n}\right) u \in L$ for all $n \in \mathbb{N}$. But $P_{e}^{n}$ is a one-dimensional projection to $\mathbb{C} \cdot q^{n}$ and therefore $\left(\mathbf{1}_{A_{\omega}} \widetilde{\otimes} P_{e}^{n}\right) u=f \otimes q^{n}$ for some $f \in A_{\omega}$ and $\left(\tau \widetilde{\otimes} \mathbf{1}_{l_{e}}\right)\left(f \otimes q^{n}\right)=\tau(f) \otimes q^{n}=0$. Therefore, $\tau(f)=0$ and $f \in I$. Since

$$
u=\lim _{k \rightarrow \infty}\left(\mathbf{1}_{A_{\omega}} \widetilde{\otimes} Q_{e}^{k}\right) u,
$$

we have the desired assertion.

It follows from Proposition 4 that $L \subset M$, because $\pi_{e}\left(f \otimes q^{n}\right)=0$ for all $f \in I$. 
Lemma 4.3. Suppose $\varphi \in{ }_{A} \operatorname{Hom}\left(M, B \widetilde{\otimes} l_{e}\right)$. Then $L \subset \operatorname{ker} \varphi$.

Proof.

Proposition 1. $I \subset \overline{A_{m} \cdot\left(A_{n} \cap A_{\omega}\right)}$ for all $m, n \in \mathbb{N}$.

Suppose $f \in I$. If $m \neq n$, choose $g \in A_{\omega}$ such that $\hat{g}\left(s_{m}\right)=1$ and $\hat{g}\left(s_{n}\right)=0$. Then $f=g f+f(e-g)$, where $g, f \in A_{n} \cap A_{\omega}$ and $f,(e-g) \in A_{m}$. If $m=n$, we have

$$
I \subset \overline{A_{m} \cdot\left(A_{m+1} \cap A_{\omega}\right)}=\overline{A_{m}^{2} \cdot\left(A_{m+1} \cap A_{\omega}\right)} \subset \overline{A_{m} \cdot\left(A_{m} \cap A_{\omega}\right)},
$$

where the equality $A_{m}=\overline{A_{m}^{2}}$ follows from the facts that $s_{m} \in \partial A$ and $A_{m}$ is projective.

Proposition 2. $f \otimes q^{n} \in \overline{A_{m} \cdot M}$ for all $f \in I, m, n \in \mathbb{N}$.

By Proposition $1 f \otimes q^{n}$ is in the closure of the linear span of the elements $g h \otimes q^{n}$, where $g \in A_{m}, h \in A_{n} \cap A_{\omega}$. But $\pi_{e}\left(h \otimes q^{n}\right)=0$ since $\hat{h}\left(s_{n}\right)=0$; i.e., $h \otimes q^{n} \in M$. Therefore, $f \otimes q^{n} \in \overline{A_{m} \cdot M}$.

Since $\varphi$ is a morphism of left $A-\widetilde{\otimes}$-modules, it follows from Proposition 2 that

$$
\varphi\left(f \otimes q^{n}\right) \in \overline{A_{m} \cdot\left(B \widetilde{\otimes} l_{e}\right)} \text { for all } f \in I, m, n \in \mathbb{N} .
$$

Proposition 3. Suppose $v \in B \widetilde{\otimes} l_{e}$ is such that for any $m \in \mathbb{N}$,

$$
v \in \overline{A_{m} \cdot\left(B \widetilde{\otimes} l_{e}\right)}
$$

Then $v=0$.

Suppose $v_{n}=\left(\mathbf{1}_{B} \widetilde{\otimes} P_{e}^{n}\right) v$. Since $P_{e}^{n}$ is a one-dimensional projection to $\mathbb{C} \cdot q^{n}$ we have $v_{n}=[f] \otimes q^{n}$ for some $f \in A$. Since $\left(\mathbf{1}_{B} \widetilde{\otimes} P_{e}^{n}\right)$ is a morphism of left $A$ - $\widetilde{\otimes}$-modules, $v_{n} \in \overline{A_{m} \cdot\left(B \widetilde{\otimes} l_{e}\right)}$ for all $m \in \mathbb{N}$. Therefore, $f \in A_{m}$ for all $m \in \mathbb{N}$; i.e., $[f]=0$. Hence, $v_{n}=0$ for all $n \in \mathbb{N}$, and

$$
v=\lim _{k \rightarrow \infty}\left(\mathbf{1}_{B} \widetilde{\otimes} Q_{e}^{k}\right) v=0 .
$$

It follows from Proposition 3 that $\varphi\left(f \otimes q^{n}\right)=0$ for all $f \in I, n \in \mathbb{N}$. Since the linear span of such elements is dense in $L$, we have the assertion of the lemma.

Theorem 4.4. Let $A$ be a weakly hereditary commutative unital $\widetilde{\otimes}$-algebra with infinite spectrum and $\left(s_{n}\right)$ a convergent sequence of points in the Shilov boundary of A such that

$$
\sigma: \widetilde{B} \rightarrow B
$$

is a retraction in the category of $A$ - $\widetilde{\otimes}$-bimodules. Then, in our notation, the map

$$
i^{*}:{ }_{A} \operatorname{Hom}\left(A_{\omega} \widetilde{\otimes} l_{e}, B \widetilde{\otimes} l_{e}\right) \rightarrow{ }_{A} \operatorname{Hom}\left(M, B \widetilde{\otimes} l_{e}\right)
$$

is surjective.

Proof. Suppose $\varphi \in{ }_{A} \operatorname{Hom}\left(M, B \widetilde{\otimes} l_{e}\right)$. Since $L \subset \operatorname{ker} \varphi, \varphi$ gives rise to a morphism

$$
\bar{\varphi}: M / L \rightarrow B \widetilde{\otimes} l_{e}
$$


Consider the commutative diagram

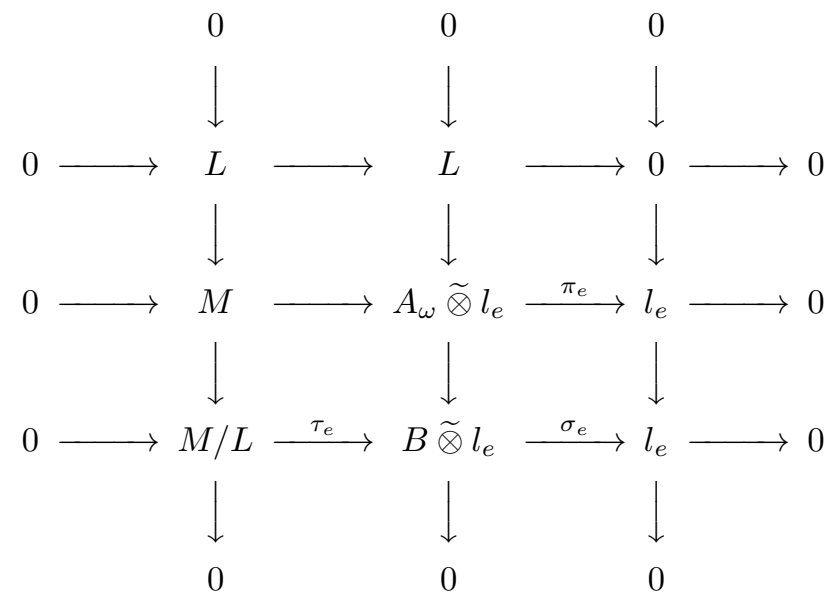

where the morphism

$$
\tau_{e}: M / L \rightarrow B \widetilde{\otimes} l_{e}
$$

is determined by the morphism

$$
\left.\tau \widetilde{\otimes} \mathbf{1}_{l_{e}}\right|_{M}: M \rightarrow B \widetilde{\otimes} l_{e}
$$

and

$$
\sigma_{e}: B \widetilde{\otimes} l_{e} \rightarrow l_{e}
$$

is determined by the condition $\sigma_{e}([f] \otimes g)=f g$. The map $\sigma_{e}$ is well-defined since for $f \in I$ and any $y \in l_{e}$ we have

$$
f y=\lim _{k \rightarrow \infty} Q_{e}^{k}(f y)=\lim _{k \rightarrow \infty} \sum_{n=1}^{k} y^{n} \hat{f}\left(s_{n}\right) q^{n}=0 .
$$

The columns and the first two rows of $\mathcal{D}$ are exact, whence the exactness of the bottom row. Consider the bilinear operator

$$
\mathcal{M}: B \times l_{e} \rightarrow\left(A_{\omega} \widetilde{\otimes} l_{e}\right) / L, \quad([f], y) \mapsto f \otimes y+L .
$$

It is well-defined since $f \otimes y \in L$ for all $f \in I$ and $y \in l_{e}$. We now show that $\mathcal{M}$ is $\widetilde{\otimes}$-bounded. We consider the cases of $\stackrel{h}{\otimes}$ - and $\stackrel{o}{\otimes}$-algebras separately.

1. $\stackrel{h}{\otimes}$-algebra. Suppose $\left(\left[f_{i j}\right]\right) \in M_{n}(B),\left(y_{i j}\right) \in M_{n}\left(l_{e}\right)$, where $n \in \mathbb{N}$. For any $\varepsilon>0$, there is a matrix $\left(f_{i j}\right) \in M_{n}\left(A_{\omega}\right)$ such that

$$
\left\|\left(f_{i j}\right)\right\| \leq\left\|\left(\left[f_{i j}\right]\right)\right\|+\varepsilon /\left\|\left(y_{i j}\right)\right\|
$$

and

$$
\begin{aligned}
\left\|\mathcal{M}_{n}^{(h)}\left(\left(\left[f_{i j}\right]\right),\left(y_{i j}\right)\right)\right\| & =\left\|\left[\left(f_{i j}\right) \odot\left(y_{i j}\right)\right]\right\| \leq\left\|\left(f_{i j}\right) \odot\left(y_{i j}\right)\right\| \\
& \leq\left\|\left(f_{i j}\right)\right\|\left\|\left(y_{i j}\right)\right\| \leq\left\|\left(\left[f_{i j}\right]\right)\right\|\left\|\left(y_{i j}\right)\right\|+\varepsilon .
\end{aligned}
$$

Therefore,

$$
\left\|\mathcal{M}_{n}^{(h)}\left(\left(\left[f_{i j}\right]\right),\left(y_{i j}\right)\right)\right\| \leq\left\|\left(\left[f_{i j}\right]\right)\right\|\left\|\left(y_{i j}\right)\right\|
$$

for all $\left(\left[f_{i j}\right]\right) \in M_{n}(B),\left(y_{i j}\right) \in M_{n}\left(l_{e}\right)$ and for any $n \in \mathbb{N}$; i.e., $\mathcal{M}$ is $\stackrel{h}{\otimes}$-bounded. 
2. $\stackrel{o}{\otimes}$-algebra. Suppose $\left(\left[f_{i j}\right]\right) \in M_{n}(B),\left(y_{i j}\right) \in M_{n}\left(l_{e}\right)$, where $n \in \mathbb{N}$. For any $\varepsilon>0$, there is a matrix $\left(f_{i j}\right) \in M_{n}\left(A_{\omega}\right)$ such that

$$
\left\|\left(f_{i j}\right)\right\| \leq\left\|\left(\left[f_{i j}\right]\right)\right\|+\varepsilon /\left\|\left(y_{i j}\right)\right\|
$$

and

$$
\begin{aligned}
\left\|\mathcal{M}_{n}^{(o)}\left(\left(\left[f_{i j}\right]\right),\left(y_{i j}\right)\right)\right\| & =\left\|\left[\left(f_{i j}\right) \otimes\left(y_{i j}\right)\right]\right\| \leq\left\|\left(f_{i j}\right) \otimes\left(y_{i j}\right)\right\| \\
& \leq\left\|\left(f_{i j}\right)\right\|\left\|\left(y_{i j}\right)\right\| \leq\left\|\left(\left[f_{i j}\right]\right)\right\|\left\|\left(y_{i j}\right)\right\|+\varepsilon .
\end{aligned}
$$

Therefore,

$$
\left\|\mathcal{M}_{n}^{(o)}\left(\left(\left[f_{i j}\right]\right),\left(y_{i j}\right)\right)\right\| \leq\left\|\left(\left[f_{i j}\right]\right)\right\|\left\|\left(y_{i j}\right)\right\|
$$

for all $\left(\left[f_{i j}\right]\right) \in M_{n}(B),\left(y_{i j}\right) \in M_{n}\left(l_{e}\right)$ and for any $n \in \mathbb{N}$; i.e., $\mathcal{M}$ is $\stackrel{o}{\otimes}$-bounded.

Therefore, there is a completely bounded operator

$$
\mu: B \widetilde{\otimes} l_{e} \rightarrow\left(A_{\omega} \widetilde{\otimes} l_{e}\right) / L,[f] \otimes y \mapsto f \otimes y+L .
$$

It is clear that $\mu$ is a morphism of left $A$ - $\widetilde{\otimes}$-modules. Since $\mathcal{D}$ is commutative with exact rows and columns, it follows that

$$
\mu\left(\tau_{e}(M / L)\right)=\mu\left(\operatorname{ker} \sigma_{e}\right) \subset M / L .
$$

Moreover, $\left.\tau_{e} \mu\right|_{\tau_{e}(M / L)}=\mathbf{1}_{\tau_{e}(M / L)}$ and $\mu \tau_{e}=\mathbf{1}_{M / L}$. Thus, we may identify $M / L$ with the closed subspace $\tau_{e}(M / L)$ in $B \widetilde{\otimes} l_{e}$.

Next we construct a completely bounded projection

$$
\chi: B \widetilde{\otimes} l_{e} \rightarrow M / L .
$$

Then we can extend $\varphi$ to $A_{\omega} \widetilde{\otimes} l_{e}$ by

$$
\widetilde{\varphi}: A_{\omega} \widetilde{\otimes} l_{e} \rightarrow B \widetilde{\otimes} l_{e}, \widetilde{\varphi}=\bar{\varphi} \circ \chi \circ\left(\tau \widetilde{\otimes} \mathbf{1}_{l_{e}}\right) .
$$

The morphism of $A$ - $\widetilde{\otimes}$-bimodules,

$$
\sigma: \widetilde{B} \rightarrow B,
$$

has a right inverse $\alpha$ in the category of $A$ - $\widetilde{\otimes}$-bimodules, and

$$
\alpha \underset{A}{\widetilde{\otimes}} \mathbf{1}_{l \infty}: B \underset{A}{\widetilde{\otimes}} l^{\infty} \rightarrow \underset{A}{\widetilde{\otimes}} l^{\infty} l^{\infty}
$$

is a morphism of left $A-\widetilde{\otimes}$-modules. We want to show that there are isomorphisms $\nu_{1}$ and $\nu_{2}$ of left $A$ - $\widetilde{\otimes}$-modules making the diagram

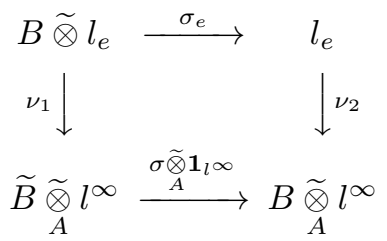

commute. Set $\nu_{2}=\tau \underset{A}{\widetilde{\otimes}} \mathbf{1}_{l \infty}$ and consider the bioperator

$$
\mathcal{S}: B \times l^{\infty} \rightarrow l_{e},([f], y) \mapsto \underset{A}{\underset{A}{\otimes} y} .
$$

It is well-defined since for all $f \in I$ and all $y \in l^{\infty}$,

$$
f \underset{A}{\otimes} y=\lim _{k \rightarrow \infty} \sum_{n=1}^{k} P_{e}^{n}(\underset{A}{\otimes} y)=\lim _{k \rightarrow \infty} \sum_{n=1}^{k} \hat{f}\left(s_{n}\right) y_{n} q^{n}=0 .
$$

We now show that $\mathcal{S}$ is $\widetilde{\otimes}$-bounded. Consider the cases of $\stackrel{h}{\otimes}$ - and $\stackrel{o}{\otimes}$-algebras separately. 
1. $\stackrel{h}{\otimes}$-algebra. Suppose $\left(\left[f_{i j}\right]\right) \in M_{n}(B),\left(y_{i j}\right) \in M_{n}\left(l^{\infty}\right)$, where $n \in \mathbb{N}$. For any $\varepsilon>0$, there is a matrix $\left(f_{i j}\right) \in M_{n}\left(A_{\omega}\right)$ such that

$$
\left\|\left(f_{i j}\right)\right\| \leq\left\|\left(\left[f_{i j}\right]\right)\right\|+\varepsilon /\left\|\left(y_{i j}\right)\right\|
$$

and

$$
\left\|\mathcal{S}_{n}^{(h)}\left(\left(\left[f_{i j}\right]\right),\left(y_{i j}\right)\right)\right\|=\left\|\left(f_{i j}\right) \bigodot_{A}\left(y_{i j}\right)\right\| \leq\left\|\left(f_{i j}\right)\right\|\left\|\left(y_{i j}\right)\right\| \leq\left\|\left(\left[f_{i j}\right]\right)\right\|\left\|\left(y_{i j}\right)\right\|+\varepsilon .
$$

Therefore,

$$
\left\|\mathcal{S}_{n}^{(h)}\left(\left(\left[f_{i j}\right]\right),\left(y_{i j}\right)\right)\right\| \leq\left\|\left(\left[f_{i j}\right]\right)\right\|\left\|\left(y_{i j}\right)\right\|
$$

for all $\left(\left[f_{i j}\right]\right) \in M_{n}(B),\left(y_{i j}\right) \in M_{n}\left(l^{\infty}\right)$ and for any $n \in \mathbb{N}$; i.e., $\mathcal{S}$ is $\stackrel{h}{\otimes}$-bounded.

2. $\stackrel{o}{\otimes}$-algebra. Suppose $\left(\left[f_{i j}\right]\right) \in M_{n}(B),\left(y_{i j}\right) \in M_{n}\left(l^{\infty}\right)$, where $n \in \mathbb{N}$. For any $\varepsilon>0$, there is a matrix $\left(f_{i j}\right) \in M_{n}\left(A_{\omega}\right)$ such that

$$
\left\|\left(f_{i j}\right)\right\| \leq\left\|\left(\left[f_{i j}\right]\right)\right\|+\varepsilon /\left\|\left(y_{i j}\right)\right\|
$$

and

$$
\left\|\mathcal{S}_{n}^{(o)}\left(\left(\left[f_{i j}\right]\right),\left(y_{i j}\right)\right)\right\|=\left\|\left(f_{i j}\right) \underset{A}{\otimes}\left(y_{i j}\right)\right\| \leq\left\|\left(f_{i j}\right)\right\|\left\|\left(y_{i j}\right)\right\| \leq\left\|\left(\left[f_{i j}\right]\right)\right\|\left\|\left(y_{i j}\right)\right\|+\varepsilon .
$$

Therefore,

$$
\left\|\mathcal{S}_{n}^{(o)}\left(\left(\left[f_{i j}\right]\right),\left(y_{i j}\right)\right)\right\| \leq\left\|\left(\left[f_{i j}\right]\right)\right\|\left\|\left(y_{i j}\right)\right\|
$$

for all $\left(\left[f_{i j}\right]\right) \in M_{n}(B),\left(y_{i j}\right) \in M_{n}\left(l^{\infty}\right)$ and for any $n \in \mathbb{N}$; i.e., $\mathcal{S}$ is $\stackrel{o}{\otimes}$-bounded.

It is clear that $\mathcal{S}$ is balanced. Therefore, there is a completely bounded operator

$$
s: B \underset{A}{\widetilde{\otimes}} l^{\infty} \rightarrow l_{e},[f] \underset{A}{\otimes} y \mapsto \underset{A}{\otimes} y .
$$

It is clear that $s$ is also a morphism of modules over $A$ in the algebraic sense and is the inverse of $\nu_{2}$; therefore $\nu_{2}$ is an isomorphism of left $A$ - $\widetilde{\otimes}$-modules.

Set

$$
\nu_{1}: B \widetilde{\otimes} l_{e} \rightarrow \underset{A}{\widetilde{B}} \underset{A}{\otimes} l^{\infty}, \nu_{1}([f] \otimes g \underset{A}{\otimes} x)=\theta([f] \otimes[g]) \underset{A}{\otimes} x
$$

where

$$
\theta: B \widetilde{\otimes} B \rightarrow \widetilde{B}
$$

is the natural projection to $\widetilde{B}=(B \widetilde{\otimes} B) / \operatorname{ker} \beta$. Since $A_{\omega} \underset{A}{\widetilde{\otimes}} l^{\infty} \cong B \underset{A}{\widetilde{\otimes}} l^{\infty}$, it suffices to show that

$$
\theta \underset{A}{\widetilde{\otimes}} \mathbf{1}_{l \infty}: B \widetilde{\otimes} B \underset{A}{\widetilde{\otimes}} l^{\infty} \rightarrow \underset{A}{\widetilde{B}} \underset{A}{\widetilde{\otimes}} l^{\infty}
$$

is an isomorphism.

We want to show that $u \underset{A}{\otimes} x=0$ for any $u \in \operatorname{ker} \beta \subset B \widetilde{\otimes} B$ and any $x \in l^{\infty}$. Since $A_{\omega} \underset{A}{\widetilde{\otimes}} l^{\infty} \cong B \underset{A}{\widetilde{\otimes}} l^{\infty}$ for all $y \in B \underset{A}{\widetilde{\otimes}} l^{\infty}$, we have

$$
y=\lim _{k \rightarrow \infty} Q_{e}^{k} y,
$$

and, therefore, for all $z \in B \widetilde{\otimes} B \underset{A}{\widetilde{\otimes}} l^{\infty}$ we have

$$
z=\lim _{k \rightarrow \infty}\left(\mathbf{1}_{B} \widetilde{\otimes} Q_{e}^{k}\right) z=\lim _{k \rightarrow \infty}\left(\mathbf{1}_{B \tilde{\otimes} B} \underset{A}{\widetilde{\otimes}} Q^{k}\right) z .
$$

Thus it suffices to show that $u \underset{A}{\otimes} e_{n}=0$ for all $n \in \mathbb{N}$. 
Let $u=\lim _{k \rightarrow \infty} \sum_{p=1}^{N_{k}}\left[f_{p}^{(k)}\right] \otimes\left[g_{p}^{(k)}\right]$, where $\left[f_{p}^{(k)}\right],\left[g_{p}^{(k)}\right] \in B$ for all $k \in \mathbb{N}, p=$ $1, \ldots, N_{k}$. For all $n, m \in \mathbb{N}$ we have

$$
\lim _{k \rightarrow \infty} \sum_{p=1}^{N_{k}} \hat{f}_{p}^{(k)}\left(s_{m}\right) \hat{g}_{p}^{(k)}\left(s_{n}\right)=0 .
$$

In particular, for all $n \in \mathbb{N}$,

$$
\lim _{k \rightarrow \infty} \sum_{p=1}^{N_{k}}\left[f_{p}^{(k)}\right] \hat{g}_{p}^{(k)}\left(s_{n}\right)=0 .
$$

Therefore, for all $n \in \mathbb{N}$,

$$
\begin{aligned}
u \underset{A}{\otimes} e_{n} & =\left(\lim _{k \rightarrow \infty} \sum_{p=1}^{N_{k}}\left[f_{p}^{(k)}\right] \otimes\left[g_{p}^{(k)}\right]\right) \underset{A}{\otimes} e_{n} \\
& =\lim _{k \rightarrow \infty} \sum_{p=1}^{N_{k}}\left[f_{p}^{(k)}\right] \otimes\left(\left[g_{p}^{(k)}\right] \underset{A}{\otimes} e_{n}\right)=\lim _{k \rightarrow \infty} \sum_{p=1}^{N_{k}}\left[f_{p}^{(k)}\right] \otimes \hat{g}_{p}^{(k)}\left(s_{n}\right) q^{n} \\
& =\lim _{k \rightarrow \infty}\left(\sum_{p=1}^{N_{k}}\left[f_{p}^{(k)}\right] \hat{g}_{p}^{(k)}\left(s_{n}\right) \otimes q^{n}\right)=\left(\lim _{k \rightarrow \infty} \sum_{p=1}^{N_{k}}\left[f_{p}^{(k)}\right] \hat{g}_{p}^{(k)}\left(s_{n}\right)\right) \otimes q^{n}=0 .
\end{aligned}
$$

Consider the operator

$$
r: \underset{A}{\widetilde{\otimes}} l^{\infty} \rightarrow B \widetilde{\otimes} B \underset{A}{\widetilde{\otimes}} l^{\infty}, v \underset{A}{\otimes} y \mapsto u \otimes_{A} y, \text { where } \theta(u)=v .
$$

Similarly to the case of $s$, one can show that $r$ is well-defined and is completely bounded. It is clear that $r$ is a morphism of left $A-\widetilde{\otimes}$-modules and is the inverse of $\theta \underset{A}{\widetilde{\otimes}} \mathbf{1}_{l \infty}$; i.e., $\theta \underset{A}{\widetilde{\otimes}} \mathbf{1}_{l \infty}$ is an isomorphism. Thus, we have proved that $\nu_{1}$ and $\nu_{2}$ are isomorphisms, and, as is easy to check, that the diagram is commutative. The morphism $\alpha \underset{A}{\widetilde{\otimes}} \mathbf{1}_{l \infty}$ is the right inverse of $\sigma \underset{A}{\widetilde{\otimes}} \mathbf{1}_{l \infty}$. The corresponding morphism

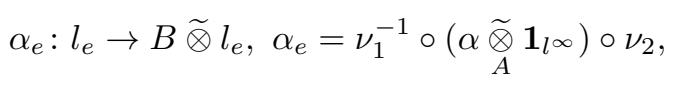

is the right inverse of $\sigma_{e}$. Therefore,

$$
\chi=\mathbf{1}_{B \tilde{\otimes} l_{e}}-\alpha_{e} \circ \sigma_{e}
$$

is a projection to $\operatorname{ker} \sigma_{e}$ and a morphism in the category of left $A-\widetilde{\otimes}$-modules. Set

$$
\widetilde{\varphi}=\bar{\varphi} \circ \chi \circ\left(\tau \widetilde{\otimes} \mathbf{1}_{l_{e}}\right) \in{ }_{A} \operatorname{Hom}\left(A_{\omega} \widetilde{\otimes} l_{e}, B \widetilde{\otimes} l_{e}\right) .
$$

Then $\left.\widetilde{\varphi}\right|_{M}=\varphi$ and the theorem is proved.

Thus we have proved that ${ }_{A} \operatorname{Ext}^{1}\left(\mathcal{Q}, B \widetilde{\otimes} l_{e}\right)=0$. To prove that ${ }_{A} \operatorname{Ext}^{1}\left(W, B \widetilde{\otimes} l_{e}\right) \neq 0$ we need to establish some preliminary results.

\section{A LOWER BOUND ON THE NORMS OF ALMOST TRIANGULAR ELEMENTS}

Consider, for $n \in \mathbb{N}$, the $n \times n$ matrix

$$
M_{0}=\left(\begin{array}{ccc}
1 & \ldots & 1 \\
& \ddots & \vdots \\
0 & & 1
\end{array}\right) .
$$

An $n \times n$ matrix $M$ is said to be $\varepsilon$-triangular if the maximum of the moduli of the entries of $M-M_{0}$ is less than or equal to $\varepsilon$. 
Definition 5.1. Suppose $E$ and $F$ are quantum spaces. An element $u \in E \widetilde{\otimes} F$ is said to be $(n, \varepsilon)$-triangular if there are continuous linear functionals $f_{1}, \ldots, f_{n} \in E^{*}$ and $g_{1}, \ldots, g_{n} \in F^{*}$ such that $\left\|f_{k}\right\| \leq 1$ and $\left\|g_{k}\right\| \leq 1$ for $k=1, \ldots, n$ and the $n \times n$ matrix $\left(f_{i} \otimes g_{j}(u)\right)$ is $\varepsilon$-triangular.

Consider the function $\Sigma$ of the natural argument defined as follows:

$$
\Sigma(n)=\frac{1}{n} \sum_{m=1}^{n-1}\left|\exp \left\{\frac{2 \pi i m}{n}\right\}-1\right|^{-1}+\frac{n+1}{2 n} .
$$

Lemma 5.2. There is a constant $C>0$ such that for all $n \in \mathbb{N}$,

$$
\Sigma(n) \geq C \log n .
$$

Proof. Since

$$
\left|\exp \left\{\frac{2 \pi i m}{n}\right\}-1\right| \leq 2 \pi \frac{m}{n}
$$

we have

$$
\Sigma(n) \geq \frac{1}{2 \pi} \sum_{m=1}^{n-1} \frac{1}{m}+\frac{n+1}{2 n} \geq \frac{1}{2 \pi} \log n .
$$

Theorem 5.3. Let $E$ and $F$ be quantum spaces and let $u \in E \widetilde{\otimes} F$ be an $(n, \varepsilon)$-triangular element. Then

$$
\|u\| \geq \Sigma(n)-n \varepsilon .
$$

Proof. Suppose $f_{k} \in E^{*}, g_{k} \in F^{*}, k=1, \ldots, n$, are such that $\left\|f_{k}\right\| \leq 1,\left\|g_{k}\right\| \leq 1$ for $k=1, \ldots, n$,

$$
\begin{array}{ll}
\left|f_{k} \otimes g_{l}(u)\right| \leq \varepsilon & \text { for } \quad k>l, \\
\left|f_{k} \otimes g_{l}(u)-1\right| \leq \varepsilon & \text { for } \quad k \leq l .
\end{array}
$$

Consider the linear functional

$$
\nu: E \otimes F \rightarrow \mathbb{C}, \nu(x \otimes y)=\frac{1}{n^{2}} \sum_{k, l, m=1}^{n} f_{k}(x) g_{l}(y) \xi^{m(l-k)} \eta_{m},
$$

where

$$
\xi=\exp \left\{\frac{2 \pi i}{n}\right\}, \quad \eta_{m}=\left\{\begin{array}{lll}
\frac{1-\xi^{m}}{\left|1-\xi^{-m}\right|} & \text { for } & m=1, \ldots, n-1, \\
1 & \text { for } & m=n .
\end{array}\right.
$$

Suppose $v \in E \otimes F$. Let $\|v\|_{\tilde{\otimes}}$ denote the norm of $v$ in $E \widetilde{\otimes} F$. For any $\varepsilon>0$ there are $N \in \mathbb{N}, x_{1}, \ldots, x_{N} \in E$, and $y_{1}, \ldots, y_{N} \in F$ such that

$$
v=\sum_{k=1}^{N} x_{k} \otimes y_{k}=\left(x_{1}, \ldots, x_{N}\right) \odot\left(\begin{array}{c}
y_{1} \\
\vdots \\
y_{N}
\end{array}\right)
$$

and

$$
\left\|\left(x_{1}, \ldots, x_{N}\right)\right\|\left\|\left(\begin{array}{c}
y_{1} \\
\vdots \\
y_{N}
\end{array}\right)\right\| \leq\|v\|_{\otimes}+\varepsilon
$$

Using the notation

$$
\left(f_{k}\left(x_{1}\right) \ldots f_{k}\left(x_{N}\right)\right)=\mathbf{f}_{k}, \quad\left(\begin{array}{c}
g_{l}\left(y_{1}\right) \\
\vdots \\
g_{l}\left(y_{N}\right)
\end{array}\right)=\mathbf{g}_{l},
$$


for $k, l=1, \ldots, n$, we have

$$
\begin{aligned}
& |\nu(v)|=\left|\frac{1}{n^{2}} \sum_{k, l, m=1}^{n} \sum_{t=1}^{N} f_{k}\left(x_{t}\right) g_{l}\left(y_{t}\right) \xi^{m(l-k)} \eta_{m}\right|=\left\|\frac{1}{n^{2}} \sum_{k, l, m=1}^{n} \mathbf{f}_{k} \mathbf{g}_{l} \xi^{m(l-k)} \eta_{m}\right\| \\
& \leq \frac{1}{n^{2}} \sum_{m=1}^{n}\left\|\sum_{k, l=1}^{n} \mathbf{f}_{k} \mathbf{g}_{l} \xi^{m(l-k)}\right\| \leq \frac{1}{n^{2}} \sum_{m=1}^{n}\left\|\sum_{k=1}^{n} \mathbf{f}_{k} \xi^{-m k}\right\| \cdot\left\|\sum_{l=1}^{n} \mathbf{g}_{l} \xi^{m l}\right\| \\
& \leq \frac{1}{n^{2}}\left(\sum_{m=1}^{n}\left\|\sum_{k=1}^{n} \mathbf{f}_{k} \xi^{-m k}\right\|^{2}\right)^{1 / 2}\left(\sum_{m=1}^{n}\left\|\sum_{l=1}^{n} \mathbf{g}_{l} \xi^{m l}\right\|^{2}\right)^{1 / 2} \\
& =\frac{1}{n^{2}}\left(\sum_{m=1}^{n}\left\|\sum_{k=1}^{n} \mathbf{f}_{k} \xi^{-m k} \cdot \sum_{l=1}^{n} \mathbf{f}_{l}^{*} \xi^{m l}\right\|\right)^{1 / 2}\left(\sum_{m=1}^{n}\left\|\sum_{k=1}^{n} \mathbf{g}_{k}^{*} \xi^{-m k} \cdot \sum_{l=1}^{n} \mathbf{g}_{l} \xi^{m l}\right\|\right)^{1 / 2} \\
& =\frac{1}{n^{2}}\left(\sum_{m=1}^{n} \sum_{k, l=1}^{n} \sum_{t=1}^{N} f_{k}\left(x_{t}\right) \overline{f_{l}\left(x_{t}\right)} \xi^{m(l-k)}\right)^{1 / 2}\left(\sum_{m=1}^{n} \sum_{k, l=1}^{n} \sum_{t=1}^{N} g_{k}\left(y_{t}\right) \overline{g_{l}\left(y_{t}\right)} \xi^{m(l-k)}\right)^{1 / 2} \\
& =\frac{1}{n}\left(\sum_{k=1}^{n} \sum_{t=1}^{N} f_{k}\left(x_{t}\right) \overline{f_{k}\left(x_{t}\right)}\right)^{1 / 2}\left(\sum_{l=1}^{n} \sum_{t=1}^{N} g_{l}\left(y_{t}\right) \overline{g_{l}\left(y_{t}\right)}\right)^{1 / 2} \\
& =\frac{1}{n}\left(\sum_{k=1}^{n}\left\|\mathbf{f}_{k}\right\|^{2}\right)^{1 / 2}\left(\sum_{l=1}^{n}\left\|\mathbf{g}_{l}\right\|^{2}\right)^{1 / 2} \leq\left\|\left(x_{1}, \ldots, x_{N}\right)\right\|\left\|\left(\begin{array}{c}
y_{1} \\
\vdots \\
y_{N}
\end{array}\right)\right\| \leq\|v\|_{\otimes}+\varepsilon .
\end{aligned}
$$

Therefore, for any $v \in E \otimes F$,

$$
|\nu(v)| \leq\|v\|_{\stackrel{h}{\otimes}} \leq\|v\|_{\otimes}^{o},
$$

and the functional $\nu$ extends to a completely bounded functional

$$
\nu: E \widetilde{\otimes} F \rightarrow \mathbb{C} \text {. }
$$

Moreover, $\|\nu\|_{c b}=\|\nu\| \leq 1$ and, for $v \in E \widetilde{\otimes} F$, we have

$$
\nu(v)=\frac{1}{n^{2}} \sum_{k, l, m=1}^{n} f_{k} \otimes g_{l}(v) \xi^{m(l-k)} \eta_{m} .
$$

Using this representation of $\nu$, for the $(n, \varepsilon)$-triangular $u$ we have an estimate

$$
|\nu(u)| \geq \Sigma(n)-n \varepsilon \text {. }
$$

Detailed computations can be found in [10]. Since $\|\nu\|_{c b} \leq 1$, we have $|\nu(u)| \leq\|u\|$ and, therefore, $\|u\| \geq \Sigma(n)-n \varepsilon$.

\section{A nontrivial group of EXtensions}

We need to show that

$$
{ }_{A} \operatorname{Ext}^{1}\left(W, B \widetilde{\otimes} l_{e}\right) \neq 0 .
$$

Consider the short admissible complex

$$
0 \leftarrow W \stackrel{\partial_{0}}{\longleftarrow} \mathcal{P} \stackrel{\partial_{1}}{\longleftarrow} A_{\omega} \widetilde{\otimes} l_{e} \leftarrow 0
$$

from Lemma 2.1. It is a projective resolution of $W$ and, since Ext does not depend on the choice of a projective resolution, ${ }_{A} \operatorname{Ext}^{1}\left(W, B \widetilde{\otimes} l_{e}\right)$ is the degree 1 cohomology group of the complex

$$
0 \rightarrow{ }_{A} \operatorname{Hom}\left(\mathcal{P}, B \widetilde{\otimes} l_{e}\right) \stackrel{\partial_{1}^{*}}{\longrightarrow}{ }_{A} \operatorname{Hom}\left(A_{\omega} \widetilde{\otimes} l_{e}, B \widetilde{\otimes} l_{e}\right) \rightarrow 0
$$


Therefore, it remains to show that $\partial_{1}^{*}$ is not surjective.

For a sequence $\left(s_{n}\right)$ of points in the Shilov boundary of $A$ we set

$$
D_{m}=\inf \left\{\|f\|: f \in A_{\omega}, \hat{f}\left(s_{1}\right)=\cdots=\hat{f}\left(s_{m}\right)=1\right\} .
$$

It is clear that $\left(D_{m}\right)$ is a nondecreasing sequence of positive numbers. Thus we can define

$$
D=\lim _{m \rightarrow \infty} D_{m}, D \in \mathbb{R} \cup\{\infty\} .
$$

For $m \in \mathbb{N}$ we set

$$
\begin{aligned}
& B_{m}^{0}=\left\{[f] \in B: \hat{f}\left(s_{1}\right)=\cdots=\hat{f}\left(s_{m}\right)=0\right\}, \\
& B_{m}^{1}=\left\{[f] \in B: \hat{f}\left(s_{1}\right)=\cdots=\hat{f}\left(s_{m}\right)=1\right\} .
\end{aligned}
$$

Let $T_{m}: B \rightarrow \mathbb{C}$ be a continuous linear functional with $\left\|T_{m}\right\|=1, T_{m}([f])=0$ for $[f] \in B_{m}^{0}$ and $T_{m}([f])=D_{m}$ for $[f] \in B_{m}^{1}$. Such a functional exists because, by the Hahn-Banach theorem, there is a continuous linear functional

$$
U_{m}: B / B_{m}^{0} \rightarrow \mathbb{C}
$$

such that $\left\|U_{m}\right\|=1, U_{m}\left([f]+B_{m}^{0}\right)=0$ for $[f] \in B_{m}^{0}$ and $U_{m}\left([f]+B_{m}^{0}\right)=D_{m}$ for $[f] \in B_{m}^{1}$. Lifting $U_{m}$ to $B$, we have a required functional $T_{m}$. Automatically, it is completely bounded and $\left\|T_{m}\right\|_{c b}=1$.

Next, for $m, n \in \mathbb{N}$, we define continuous linear functionals

$$
\begin{aligned}
& S_{n}^{m}: B \widetilde{\otimes} l_{e} \rightarrow \mathbb{C},[f] \otimes y \mapsto T_{m}([f]) y_{n}, \\
& R_{n}^{m}: B \widetilde{\otimes} l_{e} \rightarrow \mathbb{C},[f] \otimes y \mapsto \hat{f}\left(s_{m}\right) y_{n},
\end{aligned}
$$

where

$$
P_{e}^{n}(y)=y_{n} q^{n} .
$$

Since $\left\|T_{m}\right\|_{c b}=1,\left\|P_{e}^{n}\right\|_{c b}=1$, and $\left\|q^{n}\right\| \geq 1$, we have $\left\|S_{n}^{m}\right\|_{c b}=\left\|S_{n}^{m}\right\| \leq 1$ and $\left\|R_{n}^{m}\right\|_{c b}=\left\|R_{n}^{m}\right\| \leq 1$ for all $m, n \in \mathbb{N}$.

Now we state (without proof) some simple lemmas.

Lemma 6.1. Suppose $f \in A_{\omega}$ and $\hat{f}\left(s_{1}\right)=\cdots=\hat{f}\left(s_{m}\right)=1$. Then

$$
S_{n}^{m}(f \cdot u)=S_{n}^{m}(u) \text { for all } n \in \mathbb{N}, u \in B \widetilde{\otimes} l_{e} .
$$

Lemma 6.2. Suppose $f \in A_{\omega}$ and $\hat{f}\left(s_{m}\right)=1$. Then

$$
R_{n}^{m}(f \cdot u)=R_{n}^{m}(u) \text { for all } n \in \mathbb{N}, u \in B \widetilde{\otimes} l_{e} .
$$

Lemma 6.3. For any $u \in B \widetilde{\otimes} l_{e}$,

$$
\lim _{m+n \rightarrow \infty} R_{n}^{m}(u)=0 \text { and } \lim _{n \rightarrow \infty} S_{n}^{m}(u)=0 \text { for all } m \in \mathbb{N} .
$$

These results are easily checked for elementary tensors; since the linear span of such tensors is dense in $B \widetilde{\otimes} l_{e}$ and since the functionals $S_{n}^{m}$ and $R_{n}^{m}$ are bounded, these results are true for all $u \in B \widetilde{\otimes} l_{e}$.

Lemma 6.4. The sequence $\left(e \otimes q^{n}\right)$ is bounded in $A \widetilde{\otimes} l_{e}$.

Proof. For each $f \in A_{\omega}$ with $\hat{f}\left(s_{n}\right)=1$ we have

$$
\left\|q^{n}\right\|=\left\|f \underset{A}{\otimes} e_{n}\right\| \leq\|f\| .
$$

By Lemma 3.4. for any $n \in \mathbb{N}$, there is $f \in A_{\omega}$ such that $\hat{f}\left(s_{n}\right)=1$ and $\|f\| \leq$ $\|\rho\|_{c b}+1$, where $\rho$ is a coretraction of the projective maximal ideal $A_{\omega}$. Therefore, $\left\|e \otimes q^{n}\right\|=\left\|q^{n}\right\| \leq\|\rho\|_{c b}+1$.

Let $c_{0}$ denote the space of sequences converging to zero with minimal quantization. 
Lemma 6.5. The map

$$
\varkappa: l_{e} \rightarrow c_{0}, f \underset{A}{\underset{\otimes}{\otimes}} x \mapsto f \cdot x
$$

is injective. If $D<\infty$, then $\varkappa$ is an isomorphism of left $A-\widetilde{\otimes}$-modules.

Proof. The operator $\varkappa$ is well-defined and completely bounded since the bioperator

$$
A_{\omega} \times l^{\infty} \rightarrow c_{0},(f, x) \mapsto f \cdot x
$$

is $\widetilde{\otimes}$-bounded and balanced.

1) We want to show that ker $\varkappa=\{0\}$. For $f \in A_{\omega}, x \in l^{\infty}$ we have

$$
P_{e}^{n}(f \underset{A}{\otimes} x)=\hat{f}\left(s_{n}\right) x_{n} q^{n}=(\varkappa(f \underset{A}{\otimes} x))_{n} q^{n} ;
$$

hence $P_{e}^{n}(z)=(\varkappa(z))_{n} q^{n}$ for all $z \in l_{e}$. Therefore, if $\varkappa(z)=0$, then $P_{e}^{n}(z)=0$ for all $n \in \mathbb{N}$ and

$$
z=\lim _{n \rightarrow \infty} \sum_{k=1}^{n} P_{e}^{k}(z)=0 .
$$

2) Now we shall show that $\varkappa$ is an isomorphism when $D<\infty$. Suppose $N \in \mathbb{N}$ and a matrix $\left(x_{i j}\right) \in M_{N}\left(c_{0}\right)$ is such that for some $n \in \mathbb{N}$ it can be written as

$$
\left(x_{i j}\right)=\left(\sum_{k=1}^{n} \lambda_{i j}^{k} e_{k}\right),
$$

where all $\lambda_{i j}^{k} \in \mathbb{C}$. Then for

$$
\left(z_{i j}\right)=\left(\sum_{k=1}^{n} \lambda_{i j}^{k} q^{k}\right)
$$

and any $f \in A_{\omega}$ such that $\hat{f}\left(s_{1}\right)=\cdots=\hat{f}\left(s_{n}\right)=1$, we have

$$
\left(z_{i j}\right)=\left(\underset{A}{\otimes} x_{i j}\right),
$$

and, therefore, $\left\|\left(z_{i j}\right)\right\| \leq\|f\|\left\|\left(x_{i j}\right)\right\|$. Hence,

$$
\left\|\left(z_{i j}\right)\right\| \leq D_{n}\left\|\left(x_{i j}\right)\right\| \leq D\left\|\left(x_{i j}\right)\right\| .
$$

Since for any $N \in \mathbb{N}$ the set of elements $\left(\sum_{k=1}^{n} \lambda_{i j}^{k} e_{k}\right), n \in \mathbb{N}$, is dense in $M_{N}\left(c_{0}\right)$, it follows that the operator

$$
\eta: c_{0} \rightarrow l_{e}, e_{k} \mapsto q^{k},
$$

is well-defined and, for all $N \in \mathbb{N}$, for the operator

$$
\eta_{N}: M_{N}\left(c_{0}\right) \rightarrow M_{N}\left(l_{e}\right),\left(x_{i j}\right) \mapsto\left(\eta\left(x_{i j}\right)\right)
$$

we have an estimate $\left\|\eta_{N}\right\| \leq D$; i.e., $\eta$ is completely bounded. It is clear that $\eta$ is a morphism of left $A-\widetilde{\otimes}$-modules and is the inverse of $\varkappa$. Therefore, $\varkappa$ is an isomorphism of left $A$ - $\widetilde{\otimes}$-modules.

Now we can show that $\partial_{1}^{*}$ is not surjective.

Theorem 6.6. Let $A$ be a weakly hereditary commutative unital $\widetilde{\otimes}$-algebra, and $\left(s_{n}\right)$ a convergent sequence of points in $\partial A$ with a limit point $\omega \notin\left\{s_{n}: n \in \mathbb{N}\right\}$. Set

$$
\partial_{1}: A_{\omega} \widetilde{\otimes} l_{e} \rightarrow\left(A_{\omega} \widetilde{\otimes} l_{e}\right) \oplus\left(A_{\omega} \widetilde{\otimes} l^{\infty}\right), f \otimes y \mapsto(f \otimes y, f \otimes \varkappa(y)),
$$

and let

$$
\partial_{1}^{*}:{ }_{A} \operatorname{Hom}\left(\left(A_{\omega} \widetilde{\otimes} l_{e}\right) \oplus\left(A_{\omega} \widetilde{\otimes} l^{\infty}\right), B \widetilde{\otimes} l_{e}\right) \rightarrow{ }_{A} \operatorname{Hom}\left(A_{\omega} \widetilde{\otimes} l_{e}, B \widetilde{\otimes} l_{e}\right)
$$


be the corresponding map. Then the morphism

$$
\tau \widetilde{\otimes} \mathbf{1}_{l_{e}} \in{ }_{A} \operatorname{Hom}\left(A_{\omega} \widetilde{\otimes} l_{e}, B \widetilde{\otimes} l_{e}\right)
$$

is not in the image of $\partial_{1}^{*}$.

Proof. Suppose there is a morphism $\nabla \in{ }_{A} \operatorname{Hom}\left(\mathcal{P}, B \widetilde{\otimes} l_{e}\right)$ such that $\partial_{1}^{*}(\nabla)=\tau \widetilde{\otimes} \mathbf{1}_{l_{e}}$. Then we have morphisms

$$
\varphi: A \widetilde{\otimes} l_{e} \rightarrow B \widetilde{\otimes} l_{e}
$$

and

such that $\varphi \oplus \psi=\nabla$; i.e.,

$$
\psi: A_{\omega} \widetilde{\otimes} l^{\infty} \rightarrow B \widetilde{\otimes} l_{e},
$$

$$
\varphi(f \otimes y)+\psi(f \otimes \varkappa(y))=\tau(f) \otimes y
$$

for all $f \in A_{\omega}, y \in l_{e}$. We break the proof into two cases depending on the behavior of the sequence $\left(D_{m}\right)$.

1) The case $D=\infty$. For $m \in \mathbb{N}$ choose $g_{m} \in A_{\omega}$ such that

$$
\hat{g}_{m}\left(s_{1}\right)=\cdots=\hat{g}_{m}\left(s_{m}\right)=1 \text {. }
$$

Let

$$
\Phi^{(m)}: l_{e} \rightarrow c_{0}, \Phi^{(m)}(z)=\left(\Phi_{n}^{(m)}(z)\right),
$$

where

and let

$$
\Phi_{n}^{(m)}(z)=S_{n}^{m}(\varphi(e \otimes z)),
$$

$$
\Psi^{(m)}: l^{\infty} \rightarrow c_{0}, \Psi^{(m)}(x)=\left(\Psi_{n}^{(m)}(x)\right),
$$

where

$$
\Psi_{n}^{(m)}(x)=S_{n}^{m}\left(\psi\left(g_{m} \otimes x\right)\right)
$$

By Lemma 6.3, $\Phi^{(m)}$ and $\Psi^{(m)}$ are well-defined completely bounded linear operators. By Lemma 6.1, for each $n \in \mathbb{N}$ we have

$$
\Phi_{n}^{(m)}\left(q^{n}\right)+\Psi_{n}^{(m)}\left(e_{n}\right)=S_{n}^{m}\left(\tau(g) \otimes q^{n}\right)=T_{m}(\tau(g))=D_{m} .
$$

Since $\Psi^{(m)}: l^{\infty} \rightarrow c_{0}$ is a continuous linear operator, we have by the Phillips Lemma [23] that

Therefore,

$$
\Psi_{n}^{(m)}\left(e_{n}\right) \underset{n \rightarrow \infty}{\longrightarrow} 0
$$

$$
\Phi_{n}^{(m)}\left(q^{n}\right) \underset{n \rightarrow \infty}{\longrightarrow} D_{m}
$$

for all $m \in \mathbb{N}$. But, by Lemma 6.4, the sequence $\left(e \otimes q^{n}\right)$ is bounded, $\left\|S_{n}^{m}\right\| \leq 1$ for all $m, n \in \mathbb{N}$, and $D_{m} \rightarrow \infty$ for $m \rightarrow \infty$. This contradicts the continuity of $\varphi$.

2) The case $D<\infty$. For each $m \in \mathbb{N}$ we fix $h_{m} \in A_{\omega}$ such that $\hat{h}_{m}\left(s_{m}\right)=1$. Set

$$
\begin{gathered}
\Phi: l_{e} \rightarrow C_{0}\left(\mathbb{N}^{2}\right), \Phi_{m n}(z)=R_{n}^{m}(\varphi(e \otimes z)), \\
\Psi^{(m)}: l^{\infty} \rightarrow c_{0}, \Psi_{n}^{(m)}(x)=R_{n}^{m}\left(\psi\left(h_{m} \otimes x\right)\right) .
\end{gathered}
$$

By Lemma 6.3 , $\Phi$ and $\Psi^{(m)}$ are well-defined completely bounded linear operators. By Lemma 6.2. we have, for all $n, m \in \mathbb{N}$,

$$
\begin{aligned}
\Phi_{m n}\left(q^{n}\right)+\Psi_{n}^{(m)}\left(e_{n}\right) & =R_{n}^{m}\left(\varphi\left(e \otimes q^{n}\right)+\psi\left(h_{m} \otimes e_{n}\right)\right) \\
& =R_{n}^{m}\left(\varphi\left(h_{m} \otimes q^{n}\right)+\psi\left(h_{m} \otimes e_{n}\right)\right)=R_{n}^{m}\left(\tau\left(h_{m}\right) \otimes q^{n}\right)=1 .
\end{aligned}
$$

Again, by the Phillips Lemma,

$$
\lim _{n \rightarrow \infty} \Phi_{m n}\left(q^{n}\right)=1
$$

for all $m \in \mathbb{N}$. Now we need one more lemma. 
Lemma 6.7. Let $g: c_{0} \rightarrow C_{0}\left(\mathbb{N}^{2}\right)$ be a continuous linear operator such that for any $m \in \mathbb{N}$,

$$
\lim _{n \rightarrow \infty} g_{m n}\left(e_{n}\right)=1,
$$

where $g_{m n}\left(e_{n}\right)=g\left(e_{n}\right)(m, n)$. Then for any $\varepsilon>0$ and any $N \in \mathbb{N}$, there are $\left(m_{i}, n_{j}\right) \in$ $\mathbb{N}^{2}, i, j=1, \ldots, N$, and $a_{N} \in c_{0}$ such that $\left\|a_{N}\right\| \leq 2$ and

$$
\begin{array}{rll}
\left|g_{m_{i} n_{j}}\left(a_{N}\right)\right|<\varepsilon & \text { for } & i>j, \\
\left|g_{m_{i} n_{j}}\left(a_{N}\right)-1\right|<\varepsilon & \text { for } & i \leq j .
\end{array}
$$

The proof of this lemma can be found in [10; it is of a purely technical nature and is omitted here.

Using this lemma and an isomorphism between $l_{e}$ and $c_{0}$ we have $y_{N} \in l_{e}$ for each $N \in \mathbb{N}$ such that $\left\|y_{N}\right\|<K$, where $K$ is a constant independent of $N$, together with elements $\left(m_{i}, n_{j}\right) \in \mathbb{N}^{2}, i, j=1, \ldots, N$, such that

$$
\begin{aligned}
\left|\Phi_{m_{i}, n_{j}}\left(y_{N}\right)\right| & <\frac{1}{N} \text { when } i>j, \\
\left|\Phi_{m_{i}, n_{j}}\left(y_{N}\right)-1\right|<\frac{1}{N} \quad \text { when } & i \leq j .
\end{aligned}
$$

But then $\varphi\left(e \otimes y_{N}\right)$ is an $(N, 1 / N)$-triangular element in $B \widetilde{\otimes} l_{e}$ because of the continuous linear functionals

$$
[f] \mapsto \hat{f}\left(s_{m_{i}}\right), i=1, \ldots, N
$$

and

$$
y \mapsto \varkappa(y)_{n_{j}}, j=1, \ldots, N
$$

Therefore, by Theorem 5.3 ,

$$
\left\|\varphi\left(e \otimes y_{N}\right)\right\| \geq \Sigma(N)-1 \text { for all } N \in \mathbb{N} .
$$

This contradicts the continuity of $\varphi$ because the sequence $\left(y_{N}\right)$ is bounded and

$$
\Sigma(N) \underset{N \rightarrow \infty}{\longrightarrow} \infty .
$$

The theorem is proved.

To finish the proof of the main theorem we need to prove Theorem 1.4. To this end, we shall make use of the topological properties of the spectrum of a projective maximal ideal.

\section{The SPeCtrum AND A SKeleton of A PROJECTIVE IDEAL}

Let $A$ be a commutative unital $\widetilde{\otimes}$-algebra with spectrum $\Omega, I$ a projective ideal in $A$, and

$$
\rho: I \rightarrow A \widetilde{\otimes} I
$$

a coretraction of $I$. The space $C(\Omega \times \Omega)$ with minimal quantization is a left $A$ - $\widetilde{\otimes}$-module with scalar multiplication

$$
(f \cdot G)(s, t)=\hat{f}(s) G(s, t), \text { where } f \in A, G \in C(\Omega \times \Omega) .
$$

It is easy to see that the map

$$
\gamma: A \widetilde{\otimes} I \rightarrow C(\Omega \times \Omega), \gamma(f \otimes g)(s, t)=\hat{f}(s) \hat{g}(t)
$$

is a morphism of left $A-\widetilde{\otimes}$-modules. Let $\Omega_{I} \subset \Omega$ be the spectrum of $I$. 
Lemma 7.1. Suppose $s \in \Omega_{I}$ and $f_{1}, f_{2} \in I$ are such that $\hat{f}_{1}(s)=\hat{f}_{2}(s)$. Then

$$
\gamma\left(\rho\left(f_{1}\right)\right)(s, t)=\gamma\left(\rho\left(f_{2}\right)\right)(s, t) \text { for all } t \in \Omega \text {. }
$$

This assertion is obvious.

Definition 7.2. Suppose $s \in \Omega_{I}$ and $t \in \Omega$. Choose $f \in I$ with $\hat{f}(s) \neq 0$ and set

$$
F^{\rho}(s, t)=\frac{1}{\hat{f}(s)} \gamma(\rho(f))(s, t) .
$$

By Lemma 7.1, $F^{\rho}(s, t)$ does not depend on the choice of $f$, and we have a function

$$
F^{\rho}: \Omega_{I} \times \Omega \rightarrow \mathbb{C} .
$$

$F^{\rho}$ is called the skeleton of the projective ideal $I$ (corresponding to the coretraction $\rho$ ).

Now we list several simple properties of the skeleton of the projective ideal $I$ (in the case $A=C(\Omega)$ they are proved in [29]; in the general case the proof is the same).

Lemma 7.3. The skeleton $F^{\rho}$ of the projective ideal I in a $\widetilde{\otimes}$-algebra A has the following properties:

(1) $F^{\rho}$ is a continuous function on $\Omega_{I} \times \Omega$.

(2) For all $f \in I$ and $(s, t) \in \Omega_{I} \times \Omega$,

$$
\gamma(\rho(f))(s, t)=\hat{f}(s) F^{\rho}(s, t) .
$$

(3) Suppose $f \in I, s \in \Omega_{I}$ and $\hat{f}(s) \neq 0$. Then the function

$$
F_{s}^{\rho}: \Omega \rightarrow \mathbb{C}, F_{s}^{\rho}(t)=F_{s}^{\rho}(s, t)
$$

is the Gelfand transform of

$$
a_{s}=\frac{1}{\hat{f}(s)} \lim _{k \rightarrow \infty} \sum_{n=1}^{N_{k}} \hat{h}_{n}^{(k)}(s) g_{n}^{(k)} \in I, \text { where } \quad \rho(f)=\lim _{k \rightarrow \infty} \sum_{n=1}^{N_{k}} h_{n}^{(k)} \otimes g_{n}^{(k)} \text {. }
$$

(4) $F^{\rho}(s, t)=0$ for all $s \in \Omega_{I}$ and $t \in \Omega \backslash \Omega_{I}$.

(5) $F^{\rho}(s, s)=1$ for all $s \in \Omega_{I}$.

Theorem 7.4. The spectrum of the projective ideal I in a commutative unital $\widetilde{\otimes}$-algebra A is paracompact.

Proof. For the proof we need the following fact (see the proof of Th. 5.1.38 in [7]):

Lemma 7.5. Suppose that a locally compact topological space $\Omega_{I}$ admits a compactification $c \Omega_{I}$ and a continuous function

$$
G: \Omega_{I} \times c \Omega_{I} \rightarrow[0,1]
$$


such that

1) $G(s, s)=0$ for all $s \in \Omega_{I}$;

2) $G(s, t)=1$ for all $s \in \Omega_{I}, t \in c \Omega_{I} \backslash \Omega_{I}$.

Then $\Omega_{I}$ is paracompact.

Now for $c \Omega_{I}$ we take $\bar{\Omega}_{I}$. It is not difficult to check that the function

$$
G: \Omega_{I} \times \bar{\Omega}_{I} \rightarrow[0,1], G(s, t)=1-\min \left\{\left|F^{\rho}(s, t)\right|, 1\right\},
$$

where $\rho$ is a coretraction of $I$, is continuous and satisfies the conditions of Lemma 7.5. Therefore, $\Omega_{I}$ is paracompact.

\section{Nonisolated points in the Shilov BOUndary}

Let $\omega$ be a fixed nonisolated point in the Shilov boundary of a weakly hereditary commutative unital $\widetilde{\otimes}$-algebra $A$. Let

$$
\rho: A_{\omega} \rightarrow A_{\omega} \widetilde{\otimes} A_{\omega}
$$

be a coretraction of $A_{\omega}$ and $F$ the corresponding skeleton of $A_{\omega}$. Recall that for any $s \in \Omega_{I}$, the function

$$
F_{s}: \Omega \rightarrow \mathbb{C}, F_{s}(t)=F(s, t)
$$

is contained in the image of the Gelfand representation of $A$.

Let $R$ be the radical of $A$ and $\pi_{R}: A \rightarrow A / R$ the canonical projection.

Lemma 8.1. Suppose $s \in \Omega_{I}$ and $a_{s}$ is an element of $A$ such that $\hat{a}_{s}=F_{s}$. Then $\left\|\pi_{R}\left(a_{s}\right)\right\| \leq\|\rho\|_{c b}^{2}$.

Proof. We need to show that for each $s \in \Omega_{I}$ and for any $\varepsilon>0$, there is $a_{s} \in A$ such that $\hat{a}_{s}=F_{s}$ and $\left\|a_{s}\right\| \leq\|\rho\|_{c b}^{2}+\varepsilon$.

By Lemma 3.4, there is $f \in A_{\omega}$ such that $\hat{f}(s)=1$ and $\|f\| \leq\|\rho\|_{c b}+\varepsilon /\|\rho\|_{c b}$. The rest of the argument will be split into two cases for $\stackrel{h}{\otimes}$ - and $\stackrel{o}{\otimes}$-algebras.

1. $\stackrel{h}{\otimes}$-algebra. Let

$$
\rho(f)=\lim _{k \rightarrow \infty}\left(g_{i j}^{(k)}\right) \odot\left(h_{i j}^{(k)}\right),
$$

where for each $k,\left(g_{i j}^{(k)}\right) \in M_{1, N}\left(A_{\omega}\right),\left(h_{i j}^{(k)}\right) \in M_{N, 1}\left(A_{\omega}\right)$ for some $N \in \mathbb{N}$ (depending on $k$ ) and

$$
\lim _{k \rightarrow \infty}\left\|\left(g_{i j}^{(k)}\right)\right\|\left\|\left(h_{i j}^{(k)}\right)\right\|=\|\rho(f)\| .
$$

Then $F_{s}$ is the Gelfand transform of

$$
a_{s}=\lim _{k \rightarrow \infty}\left(\hat{g}_{i j}^{(k)}(s)\right)\left(h_{i j}^{(k)}\right) .
$$

Since for all $k$,

$$
\left\|\left(\hat{g}_{i j}^{(k)}(s)\right)\left(h_{i j}^{(k)}\right)\right\| \leq\left\|\left(\hat{g}_{i j}^{(k)}(s)\right)\right\|\left\|\left(h_{i j}^{(k)}\right)\right\| \leq\left\|\left(g_{i j}^{(k)}\right)\right\|\left\|\left(h_{i j}^{(k)}\right)\right\|
$$

we have

$$
\begin{aligned}
\left\|a_{s}\right\|=\lim _{k \rightarrow \infty}\left\|\left(\hat{g}_{i j}^{(k)}(s)\right)\left(h_{i j}^{(k)}\right)\right\| & \leq \lim _{k \rightarrow \infty}\left\|\left(g_{i j}^{(k)}\right)\right\|\left\|\left(h_{i j}^{(k)}\right)\right\|=\|\rho(f)\| \\
& \leq\|\rho\|_{c b} \cdot\|f\| \leq\|\rho\|_{c b}^{2}+\varepsilon
\end{aligned}
$$


2. $\stackrel{\circ}{\otimes}$-algebra. We write $\rho(f)$ as

$$
\rho(f)=\lim _{k \rightarrow \infty}\left(\alpha_{i j}^{(k)}\right)\left(\left(g_{i j}^{(k)}\right) \otimes\left(h_{i j}^{(k)}\right)\right)\left(\beta_{i j}^{(k)}\right),
$$

where for each $k,\left(\alpha_{i j}^{(k)}\right) \in M_{1, l \times p},\left(\beta_{i j}^{(k)}\right) \in M_{m \times r, 1},\left(g_{i j}^{(k)}\right) \in M_{l, m}\left(A_{\omega}\right),\left(h_{i j}^{(k)}\right) \in$ $M_{p, r}\left(A_{\omega}\right)$ for some $l, m, p, r \in \mathbb{N}$ (depending on $k$ ) and

$$
\lim _{k \rightarrow \infty}\left\|\left(\alpha_{i j}^{(k)}\right)\right\|\left\|\left(g_{i j}^{(k)}\right)\right\|\left\|\left(h_{i j}^{(k)}\right)\right\|\left\|\left(\beta_{i j}^{(k)}\right)\right\|=\|\rho(f)\| .
$$

The rest of the argument is similar to that in the $\stackrel{h}{\otimes}$-case.

Lemma 8.1 has an obvious consequence.

Corollary 8.2. The skeleton of a projective ideal is a bounded function.

The proofs of the next two lemmas are technical and are virtually the same as the proofs of the similar results for Banach algebras [11]. For this reason we omit them.

Lemma 8.3. Suppose $\left(s_{n}\right)$ is a sequence of points in $\Omega$ converging to $\omega$ such that the limits

$$
\lim _{m \rightarrow \infty} F\left(s_{m}, s_{n}\right)=\lambda\left(s_{n}\right)
$$

exist for each $n \in \mathbb{N}$ and there is a limit

$$
\lim _{n \rightarrow \infty} \lambda\left(s_{n}\right)=\mu
$$

Then $\mu=0$.

Lemma 8.4. Let $\omega$ be a nonisolated point in the Shilov boundary of A. Then there are a sequence $\left(t_{n}\right) \subset \partial A$ converging to $\omega$ and a sequence $\left(e_{n}\right) \subset A_{\omega}$ such that

$$
\hat{e}_{n}\left(t_{m}\right)=\delta_{n m} \text { for all } n, m \in \mathbb{N},
$$

and, for all $n \in \mathbb{N}, \quad\left\|e_{n}\right\|<K$ for some constant $K>0$.

Lemma 8.5. There are a sequence $\left(t_{n}\right) \subset \partial A \backslash\{\omega\}$ converging to $\omega$ and a completely bounded operator

$$
\nu: A_{\omega} \rightarrow A_{\omega} \widetilde{\otimes} A_{\omega}
$$

such that

$$
\gamma(\nu(f))\left(t_{m}, t_{n}\right)=\delta_{m n} \hat{f}\left(t_{m}\right)
$$

for all $f \in A_{\omega}$ and $m, n \in \mathbb{N}$.

Proof. Choose sequences $\left(t_{n}\right) \subset \partial A \backslash\{\omega\}$ and $\left(e_{n}\right) \subset A_{\omega}$ and a constant $K>0$ as in Lemma 8.4. Without loss of generality we may assume that $\lim _{n \rightarrow \infty}\left(\lim _{m \rightarrow \infty} F\left(t_{m}, t_{n}\right)\right)$ exists (otherwise, since $F$ is bounded, we may pass to a suitable subsequence of $\left(t_{n}\right)$ ) and equals zero (by Lemma 8.3). We may also assume that

(1) $\left|\lambda_{n}\right|<\frac{1}{2^{n}(1+n K)}$ for all $n \in \mathbb{N}$.

(2) $\left|\lambda_{m}-F\left(t_{n}, t_{m}\right)\right|<\frac{1}{2^{n+m+1}}$ for all $n \in \mathbb{N}, m=1, \ldots, n-1$.

(3) $\left|F\left(t_{m}, t_{n}\right)\right|<\frac{1}{2^{n+m+1}}$ for all $n \in \mathbb{N}, m=1, \ldots, n-1$ (for that we may choose a subsequence of $\left.\left(t_{n}\right)\right)$.

Then we have

(4) $\sum_{n=1}^{\infty} \sum_{m=1}^{n-1}\left|\lambda_{m}-F\left(t_{n}, t_{m}\right)\right|<\frac{1}{2}$ and $\sum_{n=1}^{\infty} \sum_{m=1}^{n-1}\left|F\left(t_{n}, t_{m}\right)\right|<\frac{1}{2}$. 
For each $f \in A_{\omega}$ we set

$$
f^{(k)}=f-\hat{f}\left(t_{1}\right) e_{1}-\cdots-\hat{f}\left(t_{k}\right) e_{k} .
$$

Then $\hat{f}^{(n)}\left(t_{m}\right)=\hat{f}\left(t_{m}\right)$ for $m>n$ and $\hat{f}^{(n)}\left(t_{m}\right)=0$ for $m \leq n$; moreover, for any matrix $\left(f_{i j}\right)$ with entries in $A_{\omega}$ we have

$$
\left\|\left(f_{i j}^{(k)}\right)\right\| \leq(1+k K)\left\|\left(f_{i j}\right)\right\| .
$$

Define an operator

$$
\begin{aligned}
\nu: & A_{\omega} \rightarrow A_{\omega} \widetilde{\otimes} A_{\omega}, \\
\nu(f)= & \rho(f)-\sum_{k=1}^{\infty} \lambda_{k} f^{(k)} \otimes e_{k}-\sum_{m>n}\left(F\left(t_{m}, t_{n}\right)-\lambda_{n}\right) f e_{m} \otimes e_{n} \\
& -\sum_{m<n} F\left(t_{m}, t_{n}\right) f e_{m} \otimes e_{n} .
\end{aligned}
$$

It follows from estimates (1)-(4) above that, for any $f \in A_{\omega}$, those series converge absolutely, and for any matrix $\left(f_{i j}\right)$ with entries in $A_{\omega}$ we have

$$
\begin{aligned}
\sum_{k=1}^{\infty}\left\|\left(\lambda_{k} f_{i j}^{(k)} \otimes e_{k}\right)\right\| & \leq \sum_{k=1}^{\infty}\left|\lambda_{k}\right|\left\|\left(f_{i j}^{(k)}\right)\right\|\left\|e_{k}\right\| \\
& \leq \sum_{k=1}^{\infty} \frac{1}{2^{k}(1+k K)}(1+k K)\left\|\left(f_{i j}\right)\right\| K \leq K\left\|\left(f_{i j}\right)\right\|, \\
\sum_{m>n} \|\left(\left(F\left(t_{m}, t_{n}\right)-\lambda_{n}\right)\right. & \left.f_{i j} e_{m} \otimes e_{n}\right)\left\|\leq \sum_{m>n}\left|F\left(t_{m}, t_{n}\right)-\lambda_{n}\right|\right\|\left(f_{i j}\right)\|\| e_{m}\|\| e_{n} \| \\
& \leq \sum_{m=1}^{\infty} \sum_{n=1}^{m-1}\left\|\left(f_{i j}\right)\right\| K^{2} \frac{1}{2^{n+m+1}}=\frac{1}{2}\left\|\left(f_{i j}\right)\right\| K^{2}
\end{aligned}
$$

and

$$
\sum_{m>n}\left\|\left(F\left(t_{m}, t_{n}\right) f_{i j} e_{m} \otimes e_{n}\right)\right\| \leq \sum_{n=1}^{\infty} \sum_{m=1}^{n-1}\left\|\left(f_{i j}\right)\right\| K^{2} \frac{1}{2^{n+m+1}}=\frac{1}{2}\left\|\left(f_{i j}\right)\right\| K^{2} .
$$

Hence,

$$
\left\|\left(\nu\left(f_{i j}\right)\right)\right\| \leq\left(\|\rho\|_{c b}+K+K^{2}\right)\left\|\left(f_{i j}\right)\right\|,
$$

and therefore $\nu$ is completely bounded. It is not difficult to check that

$$
\gamma(\nu(f))\left(t_{m}, t_{n}\right)=\delta_{m n} \hat{f}\left(t_{m}\right)
$$

for all $f \in A_{\omega}$ and $m, n \in \mathbb{N}$.

Now we can prove Theorem 1.4 .

Proof of Theorem 1.4. Since the spectrum of $A$ is infinite, the Shilov boundary of $A$ is also infinite [26, Th. 3.3.3]. Let $\omega$ be a nonisolated point in $\partial A$. Then there is a sequence $\left(s_{n}\right) \subset \partial A$ converging to $\omega$ with the properties mentioned in Lemma 8.5.

We need to find a morphism of $A-\widetilde{\otimes}$-bimodules which is a right inverse of

$$
\sigma:(B \widetilde{\otimes} B) / \operatorname{ker} \beta \rightarrow B,[f] \otimes[g]+\operatorname{ker} \beta \mapsto[f g] .
$$

Let $\nu: A_{\omega} \rightarrow A_{\omega} \widetilde{\otimes} A_{\omega}$ be the completely bounded operator from Lemma 8.5. Set

$$
\widetilde{\alpha}: A_{\omega} \rightarrow \widetilde{B}, \widetilde{\alpha}(f)=\theta(\tau \widetilde{\otimes} \tau(\nu(f))),
$$

where $\theta: B \widetilde{\otimes} B \rightarrow \widetilde{B}$ is the canonical projection to $(B \widetilde{\otimes} B) /$ ker $\beta$. Since

$$
\beta(\tau \widetilde{\otimes} \tau(\nu(f)))_{m n}=\gamma(\nu(f))\left(t_{m}, t_{n}\right)=\delta_{m n} \hat{f}\left(t_{n}\right),
$$


we have $\widetilde{\alpha}(f)=0$ for $f \in I$, and therefore $\widetilde{\alpha}$ gives rise to a map

$$
\alpha: B \rightarrow \widetilde{B} .
$$

We identify $\widetilde{B}=(B \widetilde{\otimes} B) / \operatorname{ker} \beta$ with a subspace in $C_{0}\left(\mathbb{N}^{2}\right)$ (via $\beta$ ) and $B$, with a subspace in $c_{0}$ (via $[f] \mapsto\left(\hat{f}\left(s_{n}\right)\right)$ ). For all $g \in A_{\omega}$ and $[f] \in B$ we have

$$
\alpha(g \cdot[f])=\alpha([g f])=\left(\delta_{m n} \hat{f}\left(t_{m}\right) \hat{g}\left(t_{m}\right)\right)_{m, n \in \mathbb{N}}=g \cdot \alpha([f]),
$$

and similarly

$$
\alpha([f] \cdot g)=\alpha([f]) \cdot g .
$$

Moreover,

$$
\|\alpha\|_{c b}=\|\widetilde{\alpha}\|_{c b} \leq\|\nu\|_{c b} ;
$$

i.e., $\alpha$ is completely bounded and is thus a morphism in the category of $A$ - $\widetilde{\otimes}$-bimodules.

It is easy to see that for any $[f] \in B$,

$$
\sigma \circ \alpha([f])=\sigma\left(\left(\delta_{m n} \hat{f}\left(t_{m}\right)\right)_{m, n \in \mathbb{N}}\right)=\left(\hat{f}\left(t_{n}\right)\right)=[f] ;
$$

i.e., $\sigma \circ \alpha=\mathbf{1}_{B}$.

Thus, $\alpha$ is a right inverse of $\sigma$ in the category of $A$ - $\widetilde{\otimes}$-bimodules.

The proof of the "quantized" version of the global dimension theorem is finished.

The author is grateful to A. Ya. Helemskii for posing the problem and to Yu. V. Selivanov for useful discussions.

\section{REFERENCES}

[1] D. P. Blecher, A completely bounded characterization of operator algebras, Math. Ann. 303 (1995), no. 2, 227-239. MR1348798 (96k:46098)

[2] D. P. Blecher and C. Le Merdy, Operator algebras and their modules-an operator space approach, London Mathematical Society Monographs. New Series, 30. Oxford Science Publications. The Clarendon Press, Oxford University Press, Oxford, 2004. MR.2111973 (2006a:46070)

[3] J. De Cannière and U. Haagerup, Multipliers of the Fourier algebras of some simple Lie groups and their discrete subgroups, Amer. J. Math. 107 (1985), no. 2, 455-500. MR784292 (86m:43002)

[4] E. G. Effros, Advances in quantized functional analysis, Proceedings of the International Congress of Mathematicians, Vols. 1, 2 (Berkeley, Calif., 1986), 906-916, Amer. Math. Soc., Providence, RI, 1987. MR934293 (89e:46064)

[5] E. G. Effros and Z.-J. Ruan, On approximation properties for operator spaces, Internat. J. Math. 1 (1990), no. 2, 163-187. MR1060634 (92g:46089)

[6] E. G. Effros and Z.-J. Ruan, Operator spaces, London Mathematical Society Monographs. New Series, 23. The Clarendon Press, Oxford University Press, New York, 2000. MR.1793753 (2002a:46082)

[7] R. Engelking, Topologia ogólna, Biblioteka Matematyczna. Tom 47. Państwowe Wydawnictwo Naukowe, Warsaw, 1975; English transl., General topology, Monografie Matematyczne, Tom 60. [Mathematical Monographs, Vol. 60] PWN-Polish Scientific Publishers, Warsaw, 1977. MR0500779 (58:18316a)

[8] A. Ya. Helemskii, The homological dimension of normed modules over Banach algebras, Mat. Sb. (N.S.) 81 (123) (1970), 430-444. (Russian) MR0262831 (41:7436)

[9] A. Ya. Helemskii, The global dimension of a functional Banach algebra is different from one, Funkcional. Anal. i Priložen. 6 (1972), no. 2, 95-96. (Russian) MR0305072 (46:4202)

[10] A. Ya. Helemskii, A certain method of computing and estimating the global homological dimension of Banach algebras, Mat. Sb. (N.S.) 87(129) (1972), 122-135; English transl., Math. USSR-Sb. 16 (1972), 125-138. MR0300087(45:9135)

[11] A. Ya. Helemskii, The lowest values of global homological dimension of functional Banach algebras, Trudy Sem. Petrovsk. 3 (1978), 223-242. (Russian)

[12] A. Ya. Helemskii, The homology of Banach and topological algebras, MGU, Moscow, 1986; English transl., Mathematics and its Applications (Soviet Series), 41, Kluwer Academic Publishers Group, Dordrecht, 1989. MR.1093462 (92d:46178)

[13] A. Ya. Helemskii, Banach and polynormed algebras: General theory, representations, homology, Nauka, Moscow, 1989; English transl. Banach and locally convex algebras, The Clarendon Press, Oxford University Press, NY, 1993. MR 1031991(91h:46001) 
[14] A. Ya. Helemskii, Wedderburn-type theorems for operator algebras and modules: Traditional and "quantized" homological approaches, Topological homology, Nova Sci. Publ., Huntington, NY, 2000, 57-92. MR1782510 (2002c:46136)

[15] A. Ya. Helemskii, Projective modules in the classical and quantum functional analysis, PIMS Distinguished Chair Lecture Notes, July-August, 2003.

[16] A. Ya. Helemskii Quantum versions of vector duality and of the exponential law in the framework of a nonmatrix approach, Mat. Sb. 197 (2006), no. 12, 133-156; English transl., Sb. Math. 197 (2006), no. 11-12, 1841-1863. MR2437084

[17] A. Ya. Helemskii, Quantum functional analysis, MCNMO, Moscow, 2009. (Russian).

[18] B. E. Johnson, Cohomology in Banach algebras, Memoirs of the American Mathematical Society, No. 127. American Mathematical Society, Providence, R.I., 1972. MR0374934 (51:11130)

[19] H. Kamovitz, Cohomology groups of commutative Banach algebras, Trans. Amer. Math. Soc. 102 (1962), 352-372. MR0170219 (30:458)

[20] V. I. Paulsen, Every completely polynomially bounded operator is similar to a contraction, J. Funct. Anal. 55 (1984), no. 1, 1-17. MR733029 (86c:47021)

[21] V. I. Paulsen, Completely bounded maps and dilations, Pitman Research Notes. 146. PitmanLongman, Wiley, NY, 1986. MR868472 (88h:46111)

[22] V. I. Paulsen, Completely bounded maps and operator algebras, Cambridge Studies in Advanced Mathematics, 78. Cambridge University Press, Cambridge, 2002. MR1976867 (2004c:46118)

[23] R. S. Phillips, On linear transformations, Trans. Amer. Math. Soc. 48 (1940), 516-541. MR0004094 $(2: 318 \mathrm{c})$

[24] J. Pisier, A polynomially bounded operator on Hilbert space which is not similar to a contraction, J. Amer. Math. Soc. 10 (1997), no. 2, 351-369. MR1415321 (97f:47002)

[25] S. Pott, An account on the global homological dimension theorem of A. Ya. Helemskii, Ann. Univ. Sarav. Ser. Math. 9 (1999), no. 3, pp. i-iv and 155-194. MR1721449 (2000i:46072)

[26] C. E. Rickart, General theory of Banach algebras, The University Series in Higher Mathematics, D. van Nostrand Co., Inc., Princeton, N.J.-Toronto-London-New York, 1960. MR0115101 (22:5903)

[27] Z.-J. Ruan, The operator amenability of $A(G)$, Amer. J. Math. 117 (1995), no. 6, 1449-1474. MR.1363075 (96m:43001)

[28] Yu. V. Selivanov, Lower bounds for homological dimensions of Banach algebras, Mat. Sb. 98 (2007), no. 9, 133-160; English transl., Sb. Math. 198 (2007), no. 9-10, 1351-1377 . MR2360795 (2008j:46035)

[29] N. V. Volosova, Description of projective ideals in quantized algebras of continuous functions on compacta, Vestnik Moskov. Univ. Ser. I Mat. Mekh. 2005, no. 5, 20-24; English transl., Moscow Univ. Math. Bull. 60 (2005), no. 5 (2006) MR2216841 (2007a:46075)

Moscow Lomonosov State University, Moscow, Russia

E-mail address: volosova_nv@mail.ru 